\title{
DOMINGO CHINEA
}

\section{JUAN CARLOS MARRERO}

\section{JUAN ROCHA}

\section{Almost contact submersions with total space a locally conformal cosymplectic manifold}

Annales de la faculté des sciences de Toulouse $6^{e}$ série, tome $4, \mathrm{n}^{\circ} 3$ (1995), p. 473-517

<http://www.numdam.org/item?id=AFST_1995_6_4_3_473_0>

(C) Université Paul Sabatier, 1995, tous droits réservés.

L'accès aux archives de la revue «Annales de la faculté des sciences de Toulouse » (http://picard.ups-tlse.fr/ annales/) implique l'accord avec les conditions générales d'utilisation (http://www.numdam.org/conditions). Toute utilisation commerciale ou impression systématique est constitutive d'une infraction pénale. Toute copie ou impression de ce fichier doit contenir la présente mention de copyright.

\section{Numdam}

Article numérisé dans le cadre du programme Numérisation de documents anciens mathématiques http://www.numdam.org/ 


\title{
Almost contact submersions with total space a locally conformal cosymplectic manifold ${ }^{(*)}$
}

\author{
Domingo Chinea ${ }^{(1)}$, Juan Carlos Marrero $^{(1)}$ \\ and JUAN ROCHA $(2)$
}

RÉSUMÉ. - Dans cet article, nous étudions les submersions métriques presque contact à espace total une variété localement conformément cosymplectique. Nous obtenons des résultats sur la minimalité des fibres sur le transfert à la base de la structure métrique presque contact, sur la structure induite sur les fibres et finalement sur l'intégrabilité de la distribution horizontale. Nous construisons le modèle local des submersions localement conformément cosymplectiques avec fibres totalement ombilicales et nous montrons que l'espace total d'une submersion localement conformément cosymplectique ne peut pas être une PC-variété (i.e., une classe particulière de variété localement conformément cosymplectique qui est feuilletée par des variétés de Hopf généralisées). Cependant, nous exhibons des exemples de submersions presque contact (qui ne sont pas des submersions riemanniennes) avec une PC-variété comme espace total. Ces exemples nous conduisent à définir les submersions $D(\sigma)$ conformément cosymplectiques. Nous en déduisons des conditions nécessaires et suffisantes sur les fibres de ce type de submersions pour qu'elles soient minimales et pour que la distribution horizontale soit intégrable. Nous étudions une classe particulière de submersion $D(\sigma)$-conformément cosymplectique qui est, dans une certaine façon, analogue à une submersion cosymplectique triviale et nous montrons que cette submersion est le modèle des submersions $D(\sigma)$-conformément cosymplectiques avec fibres totalement ombilicales et champ de vecteurs de Lee horizontal. Enfin, nous étudions les submersions $D(\sigma)$-conformément cosymplectiques avec une PC-variété comme espace total. Nous obtenons toutes les submersions $D(\sigma)$-conformément cosymplectiques à fibres totalement géodésiques et qui ont comme espace total une classe particulière de PC-variétés.

(*) Reçu le 14 juin 1993

(1) Depto. de Matemática Fundamental, Universidad de La Laguna, La Laguna, Tenerife, Canary Islands (Spain)

(2) Depto. de Matemática, Universidad de Las Palmas, Gran Canaria, Canary Island (Spain)

Supported by the "Consejería de Educación del Gobierno de Canarias" 


\title{
D. Chinea, J. C. Marrero and J. Rocha
}

\begin{abstract}
In this paper we study almost contact metric submersions with total space a locally conformal cosymplectic manifold. We obtain some results on the minimality of the fibers, transference of the almost contact metric structure to the base manifold, the induced structure on the fibers, and on the integrability of the horizontal distribution. We obtain the local model of locally conformal cosymplectic submersion with totally umbilical fibers and we show that the total space of a locally conformal cosymplectic submersion cannot be a PC-manifold (i.e. a particular class of locally conformal cosymplectic manifold which is foliated by generalized Hopf manifolds). Although, we obtain examples of almost contact submersions (which are not Riemannian submersions) with total space a PC-manifold. These examples suggest us to define the $D(\sigma)$-conformal cosymplectic submersions. Necessary and sufficient conditions for the fibers of a such submersion to be minimal and for the horizontal distribution to be completely integrable are derived. A particular class of $D(\sigma)$-conformal cosymplectic submersion which is, in certain sense, analogous to a trivial cosymplectic submersion is studied and is obtained that this submersion is the model of $D(\sigma)$-conformal cosymplectic submersion with totally umbilical fibers and horizontal Lee vector field. Finally, we study $D(\sigma)$-conformal cosymplectic submersions with total space a PC-manifold. We obtain all the $D(\sigma)$-conformal cosymplectic submersions with totally geodesic fibers and total space a particular class of PC-manifolds.
\end{abstract}

AMs Classification : 53 C 15.

KEY-WORDS : Cosymplectic manifolds, Locally conformal cosymplectic manifolds, PC-manifolds, Submersions, Almost contact metric submersions, $D(\sigma)$-conformal cosymplectic submersions.

\section{Introduction}

A smooth surjective mapping $\pi$ between almost Hermitian manifolds is said to be an almost Hermitian submersion if $\pi$ is a Riemannian submersion which is, moreover, an almost complex mapping [30].

In [30], Watson proves that if $\pi$ is an almost Hermitian submersion with total space a Kähler manifold then the base space is also a Kähler manifold, the fibers of $\pi$ are minimal submanifolds and the horizontal distribution determined by $\pi$ is completely integrable. Moreover, if the fibers of $\pi$ are totally geodesic submanifolds and the total space is complete and simply connected then the submersion $\pi$ is equivalent to a holomorphic projection. Posteriorly, in [24], De la Torre shows that if $\pi$ is an almost Hermitian submersion with total space a locally conformal Kähler (l.c.K.) manifold $M$ then the base space $M^{\prime}$ is also a l.c.K. manifold and $M^{\prime}$ is a Kähler 
manifold if and only if the fibers of $\pi$ are minimal submanifolds. More recently, in [17], we continue the study of almost Hermitian submersions with total space a l.c.K. manifold, which we call locally conformal Kähler submersions. We obtain some examples of l.c.K. submersions with total space a generalized Hopf manifold and totally geodesic fibers and derive necessary and sufficient conditions for the fibers of a l.c.K. submersion to be minimal and for that the horizontal distribution to be completely integrable. We also give relations between the Betti numbers of the total space and the base space of a l.c.K. submersion and investigate the transference of structures and some geometric properties on l.c.K. submersions with total space a generalized Hopf manifold, especially for the case when the fibers are totally geodesic submanifolds. Riemannian submersions from compact complex Hopf manifolds also have been studied by S. Dragomir in [12].

On the other hand, a submersion $\pi:(M, \varphi, \xi, \eta, g) \rightarrow\left(M^{\prime}, \varphi^{\prime}, \xi^{\prime}, \eta^{\prime}, g^{\prime}\right)$ between the almost contact metric manifolds $(M, \varphi, \xi, \eta, g)$ and $\left(M^{\prime}, \varphi^{\prime}, \xi^{\prime}, \eta^{\prime}, g^{\prime}\right)$ is said to be an almost contact submersion if $\pi$ is an almost contact mapping, i.e. $\varphi^{\prime} \circ \pi_{*}=\pi_{*} \circ \varphi$. Moreover, if $\pi$ is a Riemannian submersion then it is called an almost contact metric submersion ([5], [6] and [31]). In [5] and [6], D. Chinea proves that if $\pi$ is an almost contact metric submersion with total space a cosymplectic manifold $M$ then the base space is a cosymplectic manifold, the fibers of $\pi$ are minimal submanifolds and the horizontal distribution is completely integrable. He also shows that if $M$ is complete and simply connected and the fibers of $\pi$ are connected and totally geodesic then $M$ is a product space of a Kähler manifold with a cosymplectic manifold and $\pi$ is a projection.

Now, if $M$ is a differentiable manifold endowed with an almost contact metric structure $(\varphi, \xi, \eta, g)$, a conformal change of the metric $g$ leads to a metric which is no more compatible with the almost contact structure $(\varphi, \xi, \eta)$. This can be corrected by a convenient change of $\xi$ and $\eta$ which implies rather strong restrictions. Such a definition is given by I. Vaisman in [27]. Using this definition for the conformal change of an almost contact metric structure I. Vaisman introduces, in [27], a class of almost contact metric manifolds, called locally conformal cosymplectic manifolds. An almost contact metric manifold $(M, \varphi, \xi, \eta, g)$ is said to be locally conformal cosymplectic if the structure $(\varphi, \xi, \eta, g)$ is conformally related to a cosymplectic structure in some neighbourhood of every point of $M$ ( $\$ 1.1$ ). Recently, in [9], [11] and [16], we have continued the study of the locally conformal cosymplectic manifolds, and we have obtained some interesting examples of locally conformal cosymplectic structures on the 
real Hopf manifolds [29] and on a compact quotient of a certain solvable non-nilpotent three-dimensional Lie group. In [16], a particular class of locally conformal cosymplectic manifolds is considered, which we call PCmanifolds. A PC-manifold is a locally conformal cosymplectic manifold $(M, \varphi, \xi, \eta, g)$ with Lee 1 -form $\omega \neq 0$ at every point and such that $\omega(\xi)=0$ and the leaves of the foliation $\eta=0$ with the induced almost Hermitian structure are generalized Hopf manifolds.

In this paper, we study almost contact submersions with total space a locally conformal cosymplectic manifold. In section 1 , we recall some definitions and results on almost Hermitian, almost contact metric manifolds and almost contact metric submersions. In section 2 , we obtain some results on almost contact metric submersions with total space a locally conformal cosymplectic manifold, i.e. locally conformal cosymplectic submersions. We prove that the horizontal distribution of a locally conformal cosymplectic submersion is completely integrable. We also show that the fibers, with the induced structure, are Kähler manifolds and that the base space is a cosymplectic manifold if and only if the fibers are minimal submanifolds. We obtain a general example of locally conformal cosymplectic submersion with totally umbilical fibers and prove that, under certain conditions, this example is the local model of locally conformal cosymplectic submersion with totally umbilical fibers (corollary 2.1). Finally we show that the total space of a locally conformal cosymplectic submersion cannot be a PC-manifold.

If $(M, \varphi, \xi, \eta, g)$ is a PC-manifold then the leaves of the canonical foliation $\mathcal{F}$, given by $\eta=0, \omega=0$, have an induced $c$-Sasakian structure, where $\omega$ is the Lee 1-form of $M$ and $c=\|\omega\|$. The PC-manifold $M$ is said to be a $\mathrm{PC}(k)$-manifold $(k \in \mathbb{R})$ if every leaf $\mathrm{F}$ of the canonical foliation $\mathcal{F}$ is of constant $\varphi_{F}$-sectional curvature $\mathrm{k}$, being $\left(\varphi_{F}, \xi_{F}, \eta_{F}, g_{F}\right)$ the induced $c$ Sasakian structure on $F$ (see [16]). In section 3 , we obtain some examples of almost contact submersions (which are not Riemannian submersions) with total space a complete simply connected $\mathrm{PC}(k)$-manifold and base space a cosymplectic manifold. These submersions have totally geodesic fibers and the horizontal distribution is not completely integrable.

If $\sigma$ is a real differentiable function on an almost contact metric manifold $(M, \varphi, \xi, \eta, g)$ and $\tilde{g}$ is the Riemannian metric on $M$ given by $\tilde{g}=e^{2 \sigma} g+$ $\left(1-e^{2 \sigma}\right) \eta \otimes \eta$ then we say that $(\varphi, \xi, \eta, \tilde{g})$ is a D-conformal transformation of the structure $(\varphi, \xi, \eta, g)$. In section 4 , we define a particular class of almost contact metric manifolds called locally D-conformal cosymplectic manifolds, which we will need later, and we obtain two characterizations of 
such manifolds (propositions 4.1 and 4.2). Thus, we prove that an $(2 n+1)$ dimensional almost contact metric manifold, with $n \geq 2$, is locally $\mathrm{D}$ conformal cosymplectic if the structure $(\varphi, \xi, \eta, g)$ is D-conformally related to a cosymplectic structure in some neighbourhood of every point of $M$ (for $\operatorname{dim} M=3$ is necessary that $\mathrm{d} \sigma=\xi(\sigma) \eta)$.

The examples obtained in section 3 suggest us to consider, in section 5, a particular class of almost contact submersions with total space a globally conformal cosymplectic manifold. An almost contact submersion $\pi:(M, \varphi, \xi, \eta, g) \rightarrow\left(M^{\prime}, \varphi^{\prime}, \xi^{\prime}, \eta^{\prime}, g^{\prime}\right)$ with total space a globally conformal cosymplectic manifold $(M, \varphi, \xi, \eta, g)$ is called a $D(\sigma)$-conformal cosymplectic submersion if

$$
g_{\pi(x)}^{\prime}\left(\pi_{*}^{x} u, \pi_{*}^{x} v\right)=g_{x}(u, v)+\left(e^{2 \sigma(x)}-1\right) \eta_{x}(u) \eta_{x}(v)
$$

for all $x \in M$ and $u, v$ horizontal vectors at $x$, being $\omega=\mathrm{d} \sigma$ the Lee 1 -form of $M$. If $\operatorname{dim} M^{\prime} \geq 5$ then we deduce that $\left(M^{\prime}, \varphi^{\prime}, \xi^{\prime}, \eta^{\prime}, g^{\prime}\right)$ is a locally D-conformal cosymplectic manifold and we prove that $M^{\prime}$ is a cosymplectic manifold if and only if the Lee vector field of $M$ is vertical or equivalently if the fibers of $\pi$ are minimal submanifolds of $M$. We also obtain the corresponding results for $\operatorname{dim} M^{\prime}=3$ (corollaries 5.1 and 5.2). Finally, we show that the horizontal distribution determined by $\pi$ is completely integrable if and only if the Lee vector field of $M$ is horizontal (in this case the fibers of $\pi$, with the induced structure, are Kähler manifolds). In section 6 , we define a globally conformal cosymplectic structure on the product of a globally D-conformal cosymplectic manifold $M^{\prime}$ and a Kähler manifold $V$, such that the natural projection of $M^{\prime} \times V$ onto $M^{\prime}$ is a $D(\sigma)$-conformal cosymplectic submersion. Such submersions are called trivial $D(\sigma)$-conformal cosymplectic submersions. We prove that a trivial $D(\sigma)$-conformal cosymplectic submersion has totally umbilical fibers and the Lee vector field of the total space is horizontal. These conditions, under certain restrictions, characterize the trivial $D(\sigma)$-conformal cosymplectic submersions (theorem 6.1). In section 7 , we obtain some results on $D(\sigma)$-conformal cosymplectic submersions with total space a PC-manifold. We show that the base space of a such submersion is a locally D-conformal cosymplectic manifold with parallel Lee form or a cosymplectic manifold. We also prove that for a $D(\sigma)$ conformal cosymplectic submersion with total space a PC-manifold the Lee vector field cannot be horizontal. Consequently, the horizontal distribution is not completely integrable and the fibers, with the induced structure, are not Kähler manifolds. Finally, in section 8 we study $D(\sigma)$-conformal cosymplectic submersions with total space a PC-manifold and vertical Lee vector 
field. As main result, we show that the only $D(\sigma)$-conformal cosymplectic submersions with total space a complete simply connected $\mathrm{PC}(k)$-manifold and with connected and totally geodesic fibers are the submersions which we have obtained in section 3 (theorem 8.1).

\section{Preliminaries}

\subsection{Almost Hermitian and almost contact metric manifolds}

Let $M$ be a $C^{\infty}$ almost Hermitian manifold with metric $g$ and almost complex structure $J$. Denote by $\mathcal{X}(M)$ the Lie algebra of $C^{\infty}$ vector fields on $M$. The Kähler form $\Phi$ is given by $\Phi(X, Y)=g(X, J Y)$; the Lee form is the 1 -form $\theta$ defined by

$$
\theta(X)=\frac{1}{n-1} \delta \Phi(J X),
$$

where $\delta$ denotes the codifferential, and $\operatorname{dim} M=2 n$; and the Lee vector field is the vector field $B$ on $M$ given by $g(X, B)=\theta(X)$, for all $X \in \mathcal{X}(M)$.

Recall that $M$ is said to be Hermitian if $N=0$, being $N$ the Nijenhuis tensor of $M$; Kähler if it is Hermitian and $\mathrm{d} \Phi=0$; locally conformal Kähler (l.c.K.) if it is Hermitian, $d \Phi=\Phi \wedge \theta$ and $d \theta=0$.

Among the locally conformal Kähler manifolds, those such that the Lee form $\theta$ is parallel are called generalized Hopf manifolds ([26] and [28]).

On the other hand, an almost contact metric manifold is a quintuple $(M, \varphi, \xi, \eta, g)$ where $(M, g)$ is a Riemannian manifold, $\varphi$ a tensor field of type $(1,1)$ on $M, \xi$ a vector field and $\eta$ a 1 -form satisfying

$$
\begin{aligned}
& \varphi^{2}=-I+\eta \otimes \xi, \quad \eta(\xi)=1, \\
& g(\varphi X, \varphi Y)=g(X, Y)-\eta(X) \eta(Y),
\end{aligned}
$$

for all $X, Y \in \mathcal{X}(M), I$ being the identity transformation (the triple $(\varphi, \xi, \eta)$ is called almost contact structure and $(\varphi, \xi, \eta, g)$ is called almost contact metric structure). The fundamental 2-form $\Phi$ of an almost contact metric manifold $(M, \varphi, \xi, \eta, g)$ is defined by $\Phi(X, Y)=g(X, \varphi Y)$, and the Nijenhuis tensor of $\varphi$ is the tensor field given by

$$
N_{\varphi}(X, Y)=\varphi^{2}[X, Y]+[\varphi X, \varphi Y]-\varphi[\varphi X, Y]-\varphi[X, \varphi Y]
$$

for all $X, Y \in \mathcal{X}(M)$. 
If $\nabla$ is the Riemannian connection of $g$ and $\delta$ the codifferential, it is easy to prove that,

$$
\begin{aligned}
& \left(\nabla_{X} \Phi\right)(Y, Z)=-g\left(\left(\nabla_{X} \varphi\right) Y, Z\right) \\
& \left(\nabla_{X} \eta\right) Y=\left(\nabla_{X} \Phi\right)(\xi, \varphi Y) \\
& \operatorname{3d} \Phi(X, Y, Z)=\underset{X, Y, Z}{\mathcal{G}}\left(\nabla_{X} \Phi\right)(Y, Z) \\
& \operatorname{2d} \eta(X, Y)=\left(\nabla_{X} \Phi\right)(\xi, \varphi Y)-\left(\nabla_{Y} \Phi\right)(\xi, \varphi X) \\
& g\left(N_{\varphi}(X, Y), Z\right)= \\
& \quad=\left(\nabla_{X} \Phi\right)(\varphi Y, Z)-\left(\nabla_{\varphi Y} \Phi\right)(X, Z)+\left(\nabla_{\varphi X} \Phi\right)(Y, Z)+ \\
& \quad-\left(\nabla_{Y} \Phi\right)(\varphi X, Z)-\eta(Z)\left(\left(\nabla_{X} \Phi\right)(\xi, \varphi Y)-\left(\nabla_{Y} \Phi\right)(\xi, \varphi X)\right)+ \\
& \quad+\eta(X)\left(\nabla_{Y} \Phi\right)(\xi, \varphi Z)-\eta(Y)\left(\nabla_{X} \Phi\right)(\xi, \varphi Z) \\
& \delta \eta=-\sum_{i=1}^{n}\left(\left(\nabla_{X_{i}} \Phi\right)\left(\xi, \varphi X_{i}\right)-\left(\nabla_{\varphi X_{i}} \Phi\right)\left(\xi, X_{i}\right)\right) \\
& \delta \Phi(X)= \\
& \quad=-\sum_{i=1}^{n}\left(\left(\nabla_{X_{i}} \Phi\right)\left(X_{i}, X\right)+\left(\nabla_{\varphi X_{i}} \Phi\right)\left(\varphi X_{i}, X\right)\right)-\left(\nabla_{\xi} \Phi\right)(\xi, X),
\end{aligned}
$$

for all $X, Y, Z \in \mathcal{X}(M)$, where $\mathcal{G}$ denotes the cyclic sum over $X, Y, Z$ and $\left\{X_{1}, \ldots, X_{n}, \varphi X_{1}, \ldots, \varphi X_{n}, \xi\right\}$ is a local orthonormal $\varphi$-basis of $M$, being $\operatorname{dim} M=2 n+1$.

For an almost contact metric manifold $(M, \varphi, \xi, \eta, g)$ with $\operatorname{dim} M=$ $2 n+1 \geq 5$, the 1 -form $\omega$ defined by

$$
\omega(X)=-\frac{1}{2(n-1)}\left(\delta \Phi(\varphi X)+\left(\nabla_{\xi} \Phi\right)(\xi, \varphi X)\right)+\frac{\delta \eta}{2 n} \eta(X)
$$

is called Lee form of $M$. If $\operatorname{dim} M=3$ the 1 -form $\omega$ is defined by $\omega(X)=\left(\nabla_{\xi} \Phi\right)(\xi, \varphi X)+(\delta \eta / 2) \eta(X)$. The vector field $B$ on $M$ given by $\omega(X)=g(X, B)$, for all $X \in \mathcal{X}(M)$, is called the Lee vector field of $M$ ([10] and [11]).

An almost contact metric structure $(\varphi, \xi, \eta, g)$ is said to be [2]: normal if $N_{\varphi}+2 \mathrm{~d} \eta \otimes \xi=0$; cosymplectic if it is normal and $\mathrm{d} \Phi=0, \mathrm{~d} \eta=0$, or equivalently if $\nabla \varphi=0$. 
Let $(M, \varphi, \xi, \eta, g)$ be an almost contact metric manifold. A conformal change of the almost contact metric structure on $M$ is a change of the form

$$
\varphi^{\prime}=\varphi, \quad \xi^{\prime}=e^{-\sigma} \xi, \quad \eta^{\prime}=e^{\sigma} \eta, \quad g^{\prime}=e^{2 \sigma} g,
$$

where $\sigma$ is a real differentiable function on $M$. It is clear that $\left(\varphi^{\prime}, \xi^{\prime}, \eta^{\prime}, g^{\prime}\right)$ is also an almost contact metric structure on $M$ (see [27]).

We say that $(M, \varphi, \xi, \eta, g)$ is locally conformal cosymplectic (l.c.C.) if every point $x \in M$ has an open neighbourhood $U$ such that the almost contact metric structure $\left(\varphi^{\prime}, \xi^{\prime}, \eta^{\prime}, g^{\prime}\right)$ given in (1.9) is cosymplectic in $U$, where $\sigma: U \rightarrow \mathbb{R}$ is a real differentiable function on $U$. If the open $U$ is $M$ then $(M, \varphi, \xi, \eta, g)$ is a globally conformal cosymplectic (g.c.C.) manifold. We remark that $\omega=\mathrm{d} \sigma$ on $U$. In [11], we have proved the theorem 1.1.

THEOREM 1.1. - The following are equivalent:

(1) $(M, \varphi, \xi, \eta, g)$ is a $l(g)$.c.C. manifold;

(2) the Lee form $\omega$ is closed (exact) and

$$
\left(\nabla_{X} \varphi\right) Y=\omega(Y) \varphi X-\omega(\varphi Y) X+\Phi(X, Y) B-g(X, Y) \varphi B
$$

for all $X, Y \in \mathcal{X}(M)$;

(3) the Lee form $\omega$ is closed (exact) and

$$
\mathrm{d} \Phi=-2 \Phi \wedge \omega, \quad \mathrm{d} \eta=\eta \wedge \omega, \quad N_{\varphi}=0 .
$$

\subsection{Almost contact metric submersions}

Let $(M, g)$ and $\left(M^{\prime}, g^{\prime}\right)$ be Riemannian manifolds with $\operatorname{dim} M=m$, $\operatorname{dim} M^{\prime}=m^{\prime}$ and $m^{\prime} \leq m$. A smooth surjective mapping $\pi: M \rightarrow M^{\prime}$ is called a submersion if $\pi$ has maximal rank $m^{\prime}$. The manifolds $M$ and $M^{\prime}$ are the total space and the base space respectively of the submersion $\pi$. If $\pi:(M, g) \rightarrow\left(M^{\prime}, g^{\prime}\right)$ is a submersion then, for all $m \in M$ the subspace Ker $\pi_{*}^{m}$ is called the vertical space at $m$. Vectors on $M$ which are in the kernel of $\pi_{*}^{m}$ are tangent to the fiber $\pi_{m}=\pi^{-1}(\pi(m))$ over $m$ and are called vertical vectors at $m$. Vectors which are orthogonal to the vertical distribution are said to be horizontal. A vector field $X$ on $M$ is said to be vertical (respectively horizontal) if $X_{m}$ is vertical (respectively horizontal) for all $m \in M$. If $X$ is a vector field on $M$ then it may be written uniquely as a sum

$$
X=v X+h X,
$$

where $v X$ is a vertical vector field and $h X$ is a horizontal vector field. 
Almost contact submersions with total space a locally conformal cosymplectic manifold

A smooth vector field $X$ on $M$ is basic for the submersion $\pi: M \rightarrow M^{\prime}$ if:

(1) $X$ is horizontal, and

(2) $X$ is $\pi$-related to a vector field on $M^{\prime}$, which depends on $X$ and is denoted $X_{*}$, i.e. $\pi_{*}^{m} X_{m}=X_{* \pi(m)}$ for all $m \in M$.

There is a one-to-one relationship between basic vector fields on $M$ and vector fields on $M^{\prime}$.

Since the fibers of a submersion are regular and closed submanifolds, the vertical distribution is completely integrable. In general, the horizontal distribution is not completely integrable.

A submersion $\pi:(M, g) \rightarrow\left(M^{\prime}, g^{\prime}\right)$ between the Riemannian manifolds $(M, g)$ and $\left(M^{\prime}, g^{\prime}\right)$ is said to be a Riemannian submersion if $\left(\pi_{*}^{m}\right)_{\mid\left(\operatorname{Ker} \pi_{*}^{m}\right)^{\perp}}$ is a linear isometry for all $m \in M$ (see [19]).

Following O'Neill [19] we define the integrability tensor $A$ associated with a Riemannian submersion $\pi:(M, g) \rightarrow\left(M^{\prime}, g^{\prime}\right)$. For arbitrary vector fields $E$ and $F$ on $M$,

$$
A_{E} F=v\left(\nabla_{h E} h F\right)+h\left(\nabla_{h E} v F\right)
$$

The horizontal distribution determined by $\pi$ is completely integrable if and only if $A \equiv 0$. In fact, if $X$ and $Y$ are horizontal vector fields then $A_{X} Y=(1 / 2) v[X, Y]$ (see [19]).

Now, let $(M, \varphi, \xi, \eta, g)$ and $\left(M^{\prime}, \varphi^{\prime}, \xi^{\prime}, \eta^{\prime}, g^{\prime}\right)$ be almost contact metric manifolds. A Riemannian submersion $\pi: M \rightarrow M^{\prime}$ is called an almost contact metric submersion if $\pi$ is an almost contact mapping, i.e. $\varphi^{\prime} \circ \pi_{*}=$ $\pi_{*} \circ \varphi$.

An almost contact metric submersion $\pi: M \rightarrow M^{\prime}$ such that $M$ is member of a class $U$ of almost contact metric structure will be called $U$ submersion.

If $\pi:(M, \varphi, \xi, \eta, g) \rightarrow\left(M^{\prime}, \varphi^{\prime}, \xi^{\prime}, \eta^{\prime}, g^{\prime}\right)$ is an almost contact metric submersion then the vector field $\xi$ is horizontal and the horizontal and vertical distributions are $\varphi$-invariant [5]. In particular, the fibers of $\pi$ are invariant submanifolds of $M$ and thus they carry an induced almost Hermitian structure (for the definition and properties of invariant submanifolds of an almost contact manifold see [32]). From the above considerations we deduce that $\pi_{*} \xi= \pm \xi^{\prime}$ (we shall suppose that $\pi_{*} \xi=\xi^{\prime}$ ).

Using (1.3), (1.4), (1.5) and lemma 1 of [19], it follows [5] 
Proposition 1.1. - Let $\pi:(M, \varphi, \xi, \eta, g) \rightarrow\left(M^{\prime}, \varphi^{\prime}, \xi^{\prime}, \eta^{\prime}, g^{\prime}\right)$ be an almost contact metric submersion, and let $X, Y$ and $Z$ be basic vector fields on $M$. Then

(1) $\varphi X$ is the basic vector field associated to $\varphi^{\prime} X_{*}$;

(2) if $N_{\varphi}$ and $N_{\varphi^{\prime}}$ are the Nijenhuis tensors of $\varphi$ and $\varphi^{\prime}$, respectively, then

$$
\pi_{*}\left(N_{\varphi}(X, Y)\right)=N_{\varphi^{\prime}}\left(N_{*}, Y_{*}\right) \circ \pi
$$

(3) if $\Phi$ and $\Phi^{\prime}$ are the fundamental 2-forms of $M$ and $M^{\prime}$, respectively, then

$$
\begin{aligned}
& \Phi^{\prime}\left(X_{*}, Y_{*}\right) \circ \pi=\Phi(X, Y) \\
& \mathrm{d} \Phi^{\prime}\left(X_{*}, Y_{*}, Z_{*}\right) \circ \pi=\mathrm{d} \Phi(X, Y, Z) \\
& \eta^{\prime}\left(X_{*}\right) \circ \pi=\eta(X) \\
& \mathrm{d} \eta^{\prime}\left(X_{*}, Y_{*}\right) \circ \pi=\mathrm{d} \eta(X, Y) \\
& \left(\nabla_{X_{*}}^{\prime} \Phi^{\prime}\right)\left(Y_{*}, Z_{*}\right) \circ \pi=\left(\nabla_{X} \Phi\right)(Y, Z)
\end{aligned}
$$

being $\nabla$ and $\nabla^{\prime}$ the Riemannian connections of the metrics $g$ and $g^{\prime}$, respectively.

\section{$1.3 c$-Sasakian manifolds of constant $\varphi$-sectional curvature}

An almost contact metric manifold $(M, \varphi, \xi, \eta, g)$ is said to be $c$-Sasakian [14], with $c \in \mathbb{R}, c \neq 0$, if it is normal and $\mathrm{d} \eta=c \Phi$, where $\Phi$ is the fundamental 2-form of $M$. The manifold $M$ is said to be Sasakian if it is 1-Sasakian.

Let $(M, \varphi, \xi, \eta, g)$ be an almost contact metric manifold and $x$ a point of $M$. A plane section $P$ in the tangent space to $M$ at $x, T_{x} M$, is called a $\varphi$-section if there exists a unit vector $u$ in $T_{x} M$ orthogonal to $\xi_{x}$ such that $\left\{u, \varphi_{x} u\right\}$ is basis of $P$. Then the sectional curvature $K_{x}(u)=R_{x}\left(u, \varphi_{x} u, u, \varphi_{x} u\right)$, is called a $\varphi$-sectional curvature.

If $K_{x}(u)$ is a constant for all unit vector $u$ in $T_{x} M$ orthogonal to $\xi_{x}$ and for all point $x \in M$, then $M$ is called a space of constant $\varphi$-sectional curvature. A complete $c$-Sasakian manifold of constant $\varphi$-sectional curvature is called a $c$-Sasakian space form. 
Examples of simply connected Sasakian space forms are provided on the manifolds $S^{2 n+1}, \mathbb{R}^{2 n+1}$ and $\mathbb{R} \times C D^{n}$. In fact, the unit sphere $S^{2 n+1}$ has a Sasakian structure of constant $\varphi$-sectional curvature $k$, for all $k>-3$ ([22] and [23]); the real $(2 n+1)$-dimensional number space $\mathbb{R}^{2 n+1}$ is a Sasakian space form with $k=-3$ (see [18]); and the product manifold $\mathbb{R} \times C D^{n}$, where $C D^{n}$ is the open unit ball of $C^{n}$ with negative constant holomorphic sectional curvature $[15$, vol. II, p. 169], has a Sasakian structure of constant $\varphi$-sectional curvature $k$, for all $k<-3$ (see [23]).

Let $(M, \varphi, \xi, \eta, g)$ be a Sasakian manifold with constant $\varphi$-sectional curvature $k$. Put

$$
\varphi^{\prime}=\varphi, \quad \xi^{\prime}=c \xi, \quad \eta^{\prime}=\frac{1}{c} \eta, \quad g^{\prime}=\frac{1}{c^{2}} g
$$

where $c \in \mathbb{R}, c \neq 0$. Then $\left(M, \varphi^{\prime}, \xi^{\prime}, \eta^{\prime}, g^{\prime}\right)$ is a $c$-Sasakian manifold of constant $\varphi$-sectional curvature $k c^{2}$. We denote by $M\left(c, k c^{2}\right)$ the $c$-Sasakian manifold with this structure.

\subsection{PC-manifolds}

In [16], J. C. Marrero studies a particular class of locally conformal cosymplectic manifolds, which are called PC-manifolds. A PC-manifold is a locally conformal cosymplectic manifold $(M, \varphi, \xi, \eta, g)$ with Lee form $\omega \neq 0$ at every point and such that $\omega(\xi)=0$ and the leaves of the foliation $\eta=0$ with the induced almost Hermitian structure are generalized Hopf manifolds.

If $(M, \varphi, \xi, \eta, g)$ is a PC-manifold then $c=\|\omega\|$ is constant and

$$
\nabla \omega=-c^{2} \eta \otimes \eta, \quad \nabla_{X} B=-c^{2} \eta(X) \xi,
$$

where $B$ is the Lee vector field of $M$. Moreover, we have that [16] :

$$
\Phi=2 v \wedge u+\Psi \quad \text { with } \quad \mathrm{d} v=-c \Psi
$$

being $u=\omega / c$, and $v=-u \circ \varphi$.

We remark that a compact manifold cannot be a PC-manifold.

Let $(M, \varphi, \xi, \eta, g)$ be a PC-manifold. Denote by $\mathcal{F}$ the foliation on $M$ given by $\eta=0, u=0 . \mathcal{F}$ defines on $M$ a foliation of codimension two which is called the canonical foliation of $M$. 
Every leaf $F$ of the canonical foliation $\mathcal{F}$ has an induced $c$-Sasakian structure $\left(\varphi_{F}, \xi_{F}, \eta_{F}, g_{F}\right)$ (see [16]) which is given by

$$
\varphi_{F} X=\varphi X+\left(j^{*} v\right)(X) U_{\mid F}, \quad \xi_{F}=-V_{\mid F}, \quad \eta_{F}=-\left(j^{*} v\right), \quad g_{F}=j^{*} v,
$$

for all $X \in \mathcal{X}(F)$, where $U=B / c, V=\varphi U$, and $j: F \rightarrow M$ is the immersion of the leaf $F$ onto $M$.

A PC-manifold $M$ is called a $\mathrm{PC}(k)$-manifold $(k \in \mathbb{R})$ if every leaf $F$ of the canonical foliation $\mathcal{F}$ is of constant $\varphi_{F}$-sectional curvature $k$ (see [16]).

Now, let $\left(S, \varphi_{S}, \xi_{S}, \eta_{S}, g_{S}\right)$ be an almost contact metric manifold and $H_{c}^{2}$ the 2-dimensional hyperbolic space, i.e. $H_{c}^{2}$ is the space of 2-tuples of real numbers $(s, t)$ with the Riemannian metric given by $\mathrm{d} \tau^{2}=\mathrm{d} s^{2}+e^{-2 c s} \mathrm{~d} t^{2}$, where $c$ is a positive constant.

We define an almost contact metric structure $(\varphi, \xi, \eta, g)$ on $M=S \times H_{c}^{2}$,

$$
\begin{aligned}
& \varphi\left(X, a \frac{\partial}{\partial s}, b \frac{\partial}{\partial t}\right)=\left(\varphi_{S} X-a \xi_{S}, \eta_{S}(X) \frac{\partial}{\partial s}, 0\right) \\
& \xi=\left(0,0, e^{c s} \frac{\partial}{\partial t}\right) \\
& \eta\left(X, a \frac{\partial}{\partial s}, b \frac{\partial}{\partial t}\right)=e^{-c s} b \\
& g\left(\left(X, a \frac{\partial}{\partial s}, b \frac{\partial}{\partial t}\right),\left(X^{\prime}, a^{\prime} \frac{\partial}{\partial s}, b^{\prime} \frac{\partial}{\partial t}\right)\right)=g_{S}\left(X, X^{\prime}\right)+a a^{\prime}+e^{-2 c s} b b^{\prime}
\end{aligned}
$$

for all $X, X^{\prime} \in \mathcal{X}(S)$ and $a, a^{\prime}, b, b^{\prime}$ differentiable functions on $M$, being $(s, t)$ the coordinates on $H_{c}^{2}$. Then it follows

Proposition 1.2 [16]. - If the structure $\left(\varphi_{S}, \xi_{S}, \eta_{S}, g_{S}\right)$ on $S$ is cSasakian, then $(\varphi, \xi, \eta, g)$ is a PC-structure on $M$ with Lee 1-form $\omega=c \mathrm{~d} s$. Moreover, if $S$ is of constant $\varphi_{S}$-sectional curvature $k$, then $(\varphi, \xi, \eta, g)$ is a $\mathrm{PC}(k)$-structure on $M$.

We notice that if $\left(\varphi_{S}, \xi_{S}, \eta_{S}, g_{S}\right)$ is a $c$-Sasakian structure on $S$ then, using (1.14) and proposition 1.2, we have that the leaves of the canonical foliation of the PC-manifold $(M, \varphi, \xi, \eta, g)$ are of the form $S \times\left\{\left(s_{0}, t_{0}\right)\right\}$, with $\left(s_{0}, t_{0}\right) \in H_{c}^{2}$.

On the other hand, in [16] J. C. Marrero also proves

ThEOREM 1.2. - The universal covering space of a $(2 n+1)$-dimensional complete PC-manifold $M$ with Lee form $\omega$, is a product space $\bar{M}=S \times H_{c}^{2}$, 
Almost contact submersions with total space a locally conformal cosymplectic manifold

where $S$ is the universal covering space of an arbitrary leaf of the canonical foliation of $M, c=\|\omega\|$ and $H_{c}^{2}$ is the 2-dimensional hyperbolic space. The lift of the PC-structure to $\bar{M}$ is the PC-structure given in (1.14). Moreover, if the structure of $M$ is a $\mathrm{PC}(k)$-structure, then considering the induced $c$ Sasakian structure on $S$, we have:

(1) if $k>-3 c^{2}$ then $S$ is almost contact isometric to $S^{2 n-1}(c, k)$;

(2) if $k=-3 c^{2}$ then $S$ is almost contact isometric to $\mathbb{R}^{2 n-1}\left(c,-3 / c^{2}\right)=$ $\mathbb{R}^{2 n-1}(c)$

(3) if $k<-3 c^{2}$ then $S$ is almost contact isometric to $\left(\mathbb{R} \times C D^{n-1}\right)(c, k)$.

We remark that an isometry $F:(M, \varphi, \xi, \eta, g) \rightarrow\left(M^{\prime}, \varphi^{\prime}, \xi^{\prime}, \eta^{\prime}, g^{\prime}\right)$ between the almost contact metric manifolds $M$ and $M^{\prime}$ is said to be an almost contact isometry if $F_{*} \circ \varphi=\varphi^{\prime} \circ F_{*}$ and $F_{*} \xi=\xi^{\prime}$.

\section{Locally conformal cosymplectic submersions}

In this section we shall obtain some results on almost contact metric submersions with total space a locally conformal cosymplectic manifold.

Let $(M, \varphi, \xi, \eta, g)$ be a l.c.C. manifold, $\left(M^{\prime}, \varphi^{\prime}, \xi^{\prime}, \eta^{\prime}, g^{\prime}\right)$ an almost contact metric manifold and $\pi: M \rightarrow M^{\prime}$ be an almost contact metric submersion. We denote by $A$ the integrability tensor associated to $\pi$ and by $B$ the Lee vector field on $M$. Then we have

\section{LEMMA 2.1}

(1) The Lee vector field $B$ on $M$ is horizontal.

(2) $A_{X} Y=0$, for all horizontal vector fields $X$ and $Y$.

Proof. - Let $\nabla$ be the Riemannian connection of $(M, g)$. From (1.10) we deduce that $\nabla_{\xi} \xi=B-\eta(B) \xi$. Now, for all horizontal vector field $X$, $A_{X} X=0$ (see [19]). Then $A_{\xi} \xi=v\left(\nabla_{\xi} \xi\right)=v B=0$. Thus we obtain (1).

Let $X$ and $Y$ be horizontal vector fields. Then, from (1.10) we have

$$
\nabla_{X} \varphi Y-\varphi \nabla_{X} Y=\omega(Y) \varphi X-\omega(\varphi Y) X+\Phi(X, Y) B-g(X, Y) \varphi B .
$$

Taking vertical components in this equation, we obtain $A_{X} \varphi Y-\varphi A_{X} Y=0$. Then, since that $A_{X} Y=-A_{Y} X$ we deduce that $A_{X} \varphi Y+A_{Y} \varphi X=0$ and thus:

$$
A_{X} \xi=0 \text { and } A_{\varphi X} \varphi Y+A_{X} Y=0 .
$$


On the other hand, if $V$ is vertical and $X$ basic then [19],

$$
g\left(A_{X} \varphi Y, V\right)=-g\left(\varphi Y, A_{X} V\right)=-g\left(\varphi Y, h \nabla_{V} X\right)=g\left(Y, h \varphi \nabla_{V} X\right)
$$

Moreover, using that $h\left[\left(\nabla_{V} \varphi\right) X\right]=0$, we have that

$$
g\left(A_{X} \varphi Y, V\right)=g\left(Y, h \nabla_{V} \varphi X\right)=g\left(Y, A_{\varphi X} V\right)=-g\left(A_{\varphi X} Y, V\right)
$$

Therefore $A_{X} \varphi Y=-A_{\varphi X} Y$ and thus, from (2.1), we deduce (2).

From lemma 2.1 and using [9, corollary 2.1 and proposition 3.2], we obtain

THEOREM 2.1. - Let $\pi: M \rightarrow M^{\prime}$ be an almost contact metric submersion and $M$ a l.c.C. manifold. Then:

(1) the fibers of $\pi$, with the induced structure, are Kähler manifolds;

(2) the fibers of $\pi$ are minimal submanifolds of $M$ iff $M$ is cosymplectic;

(3) the horizontal distribution determined by $\pi$ is completely integrable.

Also, we have the following theorem.

ThEOREM 2.2. - Let $\pi: M \rightarrow M^{\prime}$ be an almost contact metric submersion and $M$ a l.c.C. manifold. Then:

(1) $M^{\prime}$ is l.c.C;

(2) $M^{\prime}$ is cosymplectic iff the fibers of $\pi$ are minimal submanifolds of $M$.

Proof. - Suppose that $\operatorname{dim} M^{\prime}=2 m^{\prime}+1 \geq 5$. Let $X$ and $Y$ be basic vector fields. From $(1.6),(1.7),(1.8)$ and proposition 1.1 , we deduce that

$$
\begin{aligned}
\omega^{\prime}\left(X_{*}\right) \circ \pi= & -\frac{1}{2\left(m^{\prime}-1\right)} \sum_{i=1}^{m^{\prime}}\left(\left(\nabla_{X_{i}} \Phi\right)\left(X_{i}, \varphi X\right)+\left(\nabla_{\varphi X_{i}} \Phi\right)\left(\varphi X_{i}, \varphi X\right)\right)+ \\
& -\frac{1}{2 m^{\prime}} \sum_{i=1}^{m^{\prime}}\left(\left(\nabla_{X_{i}} \Phi\right)\left(\xi, \varphi X_{i}\right)-\left(\nabla_{\varphi X_{i}} \Phi\right)\left(\xi, X_{i}\right)\right) \eta(X)
\end{aligned}
$$

where $\omega^{\prime}$ is the Lee 1 -form of $M^{\prime}$ and $\left\{X_{1}, \ldots, X_{m^{\prime}}, \varphi X_{1}, \ldots, \varphi X_{m^{\prime}}, \xi\right\}$ is a local orthonormal basis of the horizontal distribution of $\pi$ such that $X_{i}$ is a basic vector field for all $i=1, \ldots, m^{\prime}$. Thus, if $\omega$ is the Lee 1 -form of $M$ then, using (1.1), (1.10) and lemma 2.1, we have that

$$
\omega^{\prime}\left(X_{*}\right) \circ \pi=\omega(X), \quad \mathrm{d} \omega^{\prime}\left(X_{*}, Y_{*}\right) \circ \pi=\mathrm{d} \omega(X, Y)=0 .
$$


Almost contact submersions with total space a locally conformal cosymplectic manifold

Therefore, from (2.2), proposition 1.1 and theorem 1.1, we conclude that $\left(M^{\prime}, \varphi^{\prime}, \xi^{\prime}, \eta^{\prime}, g^{\prime}\right)$ is a l.c.C. manifold.

If $\operatorname{dim} M^{\prime}=3$ then the result is proved in a similar way.

Finally, we obtain (2) using (2.2), lemma 2.1 , theorem 2.1 and the fact that the base space of a cosymplectic submersion is a cosymplectic manifold $[5]$.

Now, let $(V, J, h)$ be a Kähler manifold and $(N, \varphi, \xi, \eta, g)$ a globally conformal cosymplectic manifold, with Lee form $\omega=-\mathrm{d}(\ln f)$, being $f>0$. On the product manifold $M=V \times N$ we consider the almost contact metric structure given by

$$
\begin{gathered}
\bar{\varphi}\left(X, X^{\prime}\right)=\left(J X, \varphi X^{\prime}\right), \quad \bar{\xi}=(0, \xi), \quad \bar{\eta}\left(X, X^{\prime}\right)=\eta\left(X^{\prime}\right) \\
\bar{g}\left(\left(X, X^{\prime}\right),\left(Y, Y^{\prime}\right)\right)=f^{2} h(X, Y)+g\left(X^{\prime}, Y^{\prime}\right)
\end{gathered}
$$

for all $X, Y \in \mathcal{X}(V)$ and $X^{\prime}, Y^{\prime} \in \mathcal{X}(N)$.

Then $(M, \bar{\varphi}, \bar{\xi}, \bar{\eta}, \bar{g})$ is also a globally conformal cosymplectic manifold with Lee form $\bar{\omega}=\pi^{*} \omega$, where $\pi: V \times N \rightarrow N$ is the projection of $M$ onto $N$. Moreover $\pi: V \times N \rightarrow N$ is an almost contact metric submersion and, since $M$ is a warped product, the fibers of $\pi$ are totally umbilical (see for instance [20]).

Next, we obtain that the preceding example is the local model of l.c.C. submersion $\pi: M \rightarrow M^{\prime}$ with totally umbilical fibers.

Let $\pi:(M, \varphi, \xi, \eta, g) \rightarrow\left(M^{\prime}, \varphi^{\prime}, \xi^{\prime}, \eta^{\prime}, g^{\prime}\right)$ be a l.c.C. submersion with connected fibers and such that $M$ is a simply connected manifold. Then, the Lee form $\omega$ is exact. Thus, if $\omega=\mathrm{d} \sigma$ we deduce that the almost contact metric structure $(\bar{\varphi}, \bar{\xi}, \bar{\eta}, \bar{g})$ on $M$ given by

$$
\bar{\varphi}=\varphi, \quad \bar{\xi}=e^{-\sigma} \xi, \quad \bar{\eta}=e^{\sigma} \eta, \quad \bar{g}=e^{2 \sigma} g,
$$

is cosymplectic. Moreover, since the Lee vector field $B$ of $(M, \varphi, \xi, \eta, g)$ is horizontal, $\sigma$ is constant on the fibers. Therefore, $\sigma$ projects to a function $\sigma^{\prime}$ on $M^{\prime}$ and the mapping $\bar{\pi}:(M, \bar{\varphi}, \bar{\xi}, \bar{\eta}, \bar{g}) \rightarrow\left(M^{\prime}, \bar{\varphi}^{\prime}, \bar{\xi}^{\prime}, \bar{\eta}^{\prime}, \bar{g}^{\prime}\right)$ defined by $\bar{\pi}(x)=\pi(x)$, is a cosymplectic submersion, where the structure $\left(\bar{\varphi}^{\prime}, \bar{\xi}^{\prime}, \bar{\eta}^{\prime}, \bar{g}^{\prime}\right)$ on $M^{\prime}$ is given by

$$
\bar{\varphi}^{\prime}=\varphi^{\prime}, \quad \bar{\xi}^{\prime}=e^{-\sigma^{\prime}} \xi^{\prime}, \quad \bar{\eta}^{\prime}=e^{\sigma^{\prime}} \eta^{\prime}, \quad \bar{g}^{\prime}=e^{2 \sigma^{\prime}} g^{\prime} .
$$




\section{Chinea, J. C. Marrero and J. Rocha}

Denote by $\nabla$ and $\bar{\nabla}$ the Riemannian connections of the metrics $g$ and $\bar{g}$ respectively. Then, if $P$ is a submanifold of $M$ and $\alpha$ (respectively $\bar{\alpha})$ is the second fundamental form of $P$ with respect to $\nabla$ (respectively $\bar{\nabla}$ ), we have

$$
\bar{\alpha}(X, Y)=\alpha(X, Y)-g(X, Y) B^{N}
$$

for all $X, Y \in \mathcal{X}(P)$, where $B^{N}$ is the normal component to $P$ of $B$.

Now, using proposition 2.2 of [9], we obtain that if the fibers of $\pi$ are totally umbilical submanifolds of $(M, g)$ then $B$ is the normal curvature vector field of such fibers. Thus, from (2.3), we deduce, in this case, that the fibers of $\bar{\pi}$ are totally geodesic submanifolds of $(M, \bar{g})$.

On the other hand, if $M$ is a complete simply connected cosymplectic manifold and $\bar{\pi}: M \rightarrow M^{\prime}$ is a cosymplectic submersion with connected and totally geodesic fibers, then $M$ is a product space of a Kähler manifold with a cosymplectic manifold and $\bar{\pi}$ is a projection, that is, $\bar{\pi}$ is a trivial cosymplectic submersion [6]. Therefore, from the above considerations, we have

Corollary 2.1. - Let $M$ be a simply connected locally conformal cosymplectic manifold, with $(M, \bar{g})$ complete, and $\pi: M \rightarrow M^{\prime}$ a l.c.C. submersion with connected and totally umbilical fibers. Then $M$ is a warped product of a Kähler manifold with a l.c.C. manifold and $\pi$ is the projection onto the l.c.C. manifold.

We also obtain the following proposition.

Proposition 2.1. - If $\pi: M \rightarrow M^{\prime}$ is an almost contact metric submersion, then $M$ is not a PC-manifold.

Proof.- We suppose that $(M, \varphi, \xi, \eta, g)$ is a PC-manifold with Lee vector field $B$. Then by lemma 2.1 , the vector fields $B$ and $\varphi B$ are horizontal. Denote by $\Psi$ the 2-form on $M$ given by $\Psi=\Phi-2 v \wedge u$, where $\Phi$ is the fundamental 2-form of the structure $(\varphi, \xi, \eta, g), u$ is the unit Lee 1 -form and $v=-u \circ \varphi$. Then, if $W$ and $W^{\prime}$ are vertical vector fields and $c=\|\omega\|$, from (1.12), we have

$$
\Phi\left(W, W^{\prime}\right)=\Psi\left(W, W^{\prime}\right)=-\frac{1}{c} \mathrm{~d} v\left(W, W^{\prime}\right)=0,
$$

which is a contradiction. 
Almost contact submersions with total space a locally conformal cosymplectic manifold

\section{Examples of almost contact submersions with total space a PC-manifold}

In this section, we obtain some examples of almost contact submersions with total space a PC-manifold which are not Riemannian submersions (corollary 3.2). First, we recall some results on almost contact metric submersions of type II ([31], [25], [17] and [21']).

Definition 3.1 ([31] and [25]). - Let $(S, \varphi, \xi, \eta, g)$ be an almost contact metric manifold and let $\bar{\pi}: S \rightarrow V$ be a Riemannian submersion onto the almost Hermitian manifold $(V, J, h)$ which satisfies :

$$
J_{\bar{\pi}(x)}\left(\bar{\pi}_{*}^{x} v\right)=\bar{\pi}_{*}^{x}\left(\varphi_{x} v\right)
$$

for all $x \in S$ and $v \in T_{x} S$. Then, $\bar{\pi}$ is said to be an almost contact metric submersion of type II.

In [31] (also [25]) the author proves

Proposition 3.1. - If $\bar{\pi}:(S, \varphi, \xi, \eta, g) \rightarrow(V, J, h)$ is an almost contact metric submersion of type II, then :

(1) $\xi$ is a vertical vector field;

(2) the horizontal and vertical distributions determined by $\bar{\pi}$ are $\varphi$ invariant.

We also have $\left[21^{\prime}\right]$

Proposition 3.2. - Let $\bar{\pi}:(S, \varphi, \xi, \eta, g) \rightarrow(V, J, h)$ be an almost contact metric submersion of type II such that $(S, \varphi, \xi, \eta, g)$ is a c-Sasakian manifold. Then:

(1) $(V, J, h)$ is a Kähler manifold;

(2) $\bar{A}_{X} Y=-c \Phi(X, Y) \xi$, where $\bar{A}$ is the integrability tensor of $\bar{\pi}, \Phi$ is the fundamental 2-form of $S$ and $X, Y$ are horizontal vector fields.

Consequently, we deduce [21']

Corollary 3.1. - If $\bar{\pi}:(S, \varphi, \xi, \eta, g) \rightarrow(V, J, h)$ is an almost contact metric submersion of type II and $(S, \varphi, \xi, \eta, g)$ is a c-Sasakian manifold, then the horizontal distribution of $\bar{\pi}$ is not completely integrable. 
Next, we obtain some examples of almost contact metric submersions of type II with total space a $c$-Sasakian manifold [21'].

Let $c$ be a real number, $c \neq 0$ and $k>-3 c^{2}$. Denote by $\bar{\pi}_{1}(c, k, n)$ the Hopf fibration of the $c$-Sasakian manifold $S^{2 n+1}(c, k)$ onto the $n$ dimensional complex projective space $P_{n}\left(C^{n+1}\right)\left(k+3 c^{2}\right)$ of positive constant holomorphic sectional curvature $k+3 c^{2}$ [15, vol. II, p. 169]. Analogously if $k<-3 c^{2}$ denote by $\bar{\pi}_{3}(c, k, n)$ the natural projection of the $c$-Sasakian manifold $\left(\mathbb{R} \times C D^{n}\right)(c, k)$ onto the $n$-dimensional open unit ball $C D^{n}\left(k+3 c^{2}\right)$ in $C^{n}$ of negative constant holomorphic sectional curvature $k+3 c^{2}\left[15\right.$, vol. II, p. 169]. Finally, let $\bar{\pi}_{2}(c, n, m): \mathbf{R}^{2 n+1}(c) \rightarrow C^{m}(0) \simeq$ $\mathbb{R}^{2 m}(0)$, with $m \leq n$, be the canonical projection of $R^{2 n+1}(c)$ onto the flat Kähler space $C^{m}(0)$. Then

Proposition $3.3\left[21^{\prime}\right]$. - For all $k>-3 c^{2}$ (respectively $k=-3 c^{2}$ and $k<-3 c^{2}$ ) the submersion $\bar{\pi}_{1}(c, k, n)$ (respectively $\bar{\pi}_{2}(c, n, m)$ and $\left.\bar{\pi}_{3}(c, k, n)\right)$ is an almost contact metric submersion of type II with total space a c-Sasakian manifold of constant $\varphi$-sectional curvature $k$ and with totally geodesic fibers.

Now, let $\bar{\pi}:\left(S, \varphi_{S}, \xi_{S}, \eta_{S}, g_{S}\right) \rightarrow(V, J, h)$ be an almost contact metric submersion of type II with total space a $c$-Sasakian manifold $S$. Consider on the product manifold $M^{\prime}=V \times \mathbb{R}$ the almost contact metric structure $\left(\varphi^{\prime}, \xi^{\prime}, \eta^{\prime}, g^{\prime}\right)$ defined by

$$
\begin{aligned}
& \varphi^{\prime}\left(X, a \frac{\partial}{\partial t}\right)=(J X, 0), \quad \xi^{\prime}=\left(0, \frac{\partial}{\partial t}\right), \quad \eta^{\prime}\left(X, a \frac{\partial}{\partial t}\right)=a \\
& g^{\prime}\left(\left(X, a \frac{\partial}{\partial t}\right),\left(Y, b \frac{\partial}{\partial t}\right)\right)=h(X, Y)+a b
\end{aligned}
$$

for all $X, Y$ vector fields on $V$ and $a, b$ differentiable functions on $M^{\prime}$, where $t$ is the usual coordinate on $\mathbb{R}$.

Then, since $(V, J, h)$ is a Kähler manifold, we have that $\left(M^{\prime}, \varphi^{\prime}, \xi^{\prime}, \eta^{\prime}, g^{\prime}\right)$ is a cosymplectic manifold (see for instance [3] and [7]). Moreover, if we denote by $(\varphi, \xi, \eta, g)$ the almost contact metric structure on $M=S \times H_{c}^{2}$ given by (1.14) and by $\pi$ the mapping of $M$ onto $M^{\prime}$ defined by

$$
\pi(x,(s, t))=(\bar{\pi}(x), t)
$$

being $(s, t)$ the usual coordinates on $H_{c}^{2}$ and $x \in S$, then we deduce 
Almost contact submersions with total space a locally conformal cosymplectic manifold

Proposition 3.4. - The mapping $\pi:(M, \varphi, \xi, \eta, g) \rightarrow\left(M^{\prime}, \varphi^{\prime}, \xi^{\prime}, \eta^{\prime}, g^{\prime}\right)$ is a submersion of the PC-manifold $M$ onto the cosymplectic manifold $M^{\prime}$ which satisfies:

(1) $\pi$ is an almost contact mapping, i.e. $\pi_{*} \circ \varphi=\varphi^{\prime} \circ \pi_{*}$;

(2) for all $y=(x,(s, t)) \in M$ and for all $u$, $v$ horizontal vectors at $y$ :

$$
g_{\pi(y)}^{\prime}\left(\pi_{*}^{y} u, \pi_{*}^{y} v\right)=g_{y}(u, v)+\left(e^{2 c s}-1\right) \eta_{y}(u) \eta_{y}(v)
$$

(3) the fibers of $\pi$ are totally geodesic submanifolds of $M$ if and only if the fibers of $\bar{\pi}$ are totally geodesic submanifolds of $S$;

(4) The horizontal distribution of $\pi$ is not completely integrable.

Proof. - Let $y$ be a point of $M, y=(x,(s, t))$ with $x \in S$ and $(s, t) \in H_{c}^{2}$, and $H_{y}(M)$ (respectively $\bar{H}_{x}(S)$ ) the subspace of horizontal vectors at $y$ (respectively $x$ ) of the submersion $\pi$ (respectively $\bar{\pi}$ ). Then,

$$
H_{y}(M)=\bar{H}_{x}(S) \oplus\left\langle\frac{\partial}{\partial t_{\mid t}}\right\rangle .
$$

Now, from definition 3.1, proposition 3.1, corollary 3.1 and using (1.14), (3.1) and (3.4) we deduce (1), (2) and (4).

On the other hand, if $F_{y}$ (respectively $\bar{F}_{x}$ ) is the fiber of $\pi$ (respectively $\bar{\pi}$ ) over $y$ (respectively $x$ ) then $F_{y}$ is the submanifold $\bar{F}_{x} \times \mathbb{R} \times\{t\}$. Moreover, the induced metric by $g$ on $F_{y}$ is the product metric. Consequently, $F_{y}$ is a totally geodesic submanifold of $M$ if and only if $\bar{F}_{x}$ is a totally geodesic submanifold of $S$.

Now, let $c$ be a real number, $c \neq 0$ and let the mappings $\pi_{1}(c, k, n)$, $\pi_{2}(c, n, m)$ and $\pi_{3}(c, k, n)$ :

$$
\begin{aligned}
\forall k>-3 c^{2}, & \pi_{1}(c, k, n): S^{2 n+1}(c, k) \times H_{c}^{2} \longrightarrow P_{n}\left(C^{n+1}\right)\left(k+3 c^{2}\right) \times \mathbb{R} \\
m \leq n, & \pi_{2}(c, n, m): \mathbb{R}^{2 n+1}(c) \times H_{c}^{2} \longrightarrow \mathbb{R}^{2 m}(0) \times \mathbb{R} \simeq C^{m}(0) \times \mathbb{R} \\
\forall k<-3 c^{2}, & \pi_{3}(c, k, n):\left(\mathbb{R} \times C D^{n}\right)(c, k) \times H_{c}^{2} \longrightarrow C D^{n}\left(k+3 c^{2}\right) \times \mathbb{R}
\end{aligned}
$$

induced by $\bar{\pi}_{1}(c, k, n), \bar{\pi}_{2}(c, n, m)$ and $\bar{\pi}_{3}(c, k, n)$, by using (3.2). Then, from propositions $3.3,3.4$ and 1.2 , we obtain the main result of this section 


\section{Chinea, J. C. Marrero and J. Rocha}

COROLLARY 3.2. - For all $k>-3 c^{2}$ (respectively $k=-3 c^{2}, k<-3 c^{2}$ ) the submersion $\pi_{1}(c, k, n)$ (respectively $\pi_{2}(c, n, m)$ and $\pi_{3}(c, k, n)$ ) is an almost contact submersion which satisfies (3.3) and such that the total space is a complete simply connected $\mathrm{PC}(k)$-manifold, the base space is a complete simply connected cosymplectic manifold, the fibers are totally geodesic submanifolds and the horizontal distribution is not completely integrable.

\section{Locally D-conformal cosymplectic manifolds}

In this section, we study a particular class of almost contact metric manifolds, which we call locally $\mathrm{D}$-conformal cosymplectic manifolds. We shall use these manifolds in the following sections.

Let $(M, \varphi, \xi, \eta, g)$ be an almost contact metric manifold and $c$ a positive number. Define an almost contact metric structure $(\tilde{\varphi}, \tilde{\xi}, \tilde{\eta}, \tilde{g})$ on $M$ by

$$
\tilde{\varphi}=\varphi, \quad \tilde{\xi}=\frac{1}{c} \xi, \quad \tilde{\eta}=c \eta, \quad \tilde{g}=c g+\left(c^{2}-c\right) \eta \otimes \eta
$$

A such deformation is called D-homothetic (see for instance [1] and [22]).

In general, if $\lambda$ and $\mu$ are differentiable functions on $M$ such that $\lambda>0$ and $\lambda+\mu>0$ then we can define an almost contact metric structure $(\tilde{\varphi}, \tilde{\xi}, \tilde{\eta}, \tilde{g})$ on $M$ by

$$
\tilde{\varphi}=\varphi, \quad \tilde{\xi}=\frac{1}{\sqrt{\lambda+\mu}} \xi, \quad \tilde{\eta}=(\sqrt{\lambda+\mu}) \eta, \quad \tilde{g}=\lambda g+\mu \eta \otimes \eta
$$

We call this deformation a $D(\lambda, \mu)$-conformal transformation [1].

If $\mu=1-\lambda$ then we say that $(\tilde{\varphi}, \tilde{\xi}, \tilde{\eta}, \tilde{g})$ is a D-conformal transformation of the structure $(\varphi, \xi, \eta, g)$.

If $\lambda=1$ then $\mu=e^{2 \sigma}-1$, being $\sigma$ a differentiable function on $M$. In this case, we say that $(\tilde{\varphi}, \tilde{\xi}, \tilde{\eta}, \tilde{g})$ is a $D(\sigma)$-conformal transformation of the structure $(\varphi, \xi, \eta, g)$. 
Almost contact submersions with total space a locally conformal cosymplectic manifold

Definition 4.1. - An almost contact metric manifold $(M, \varphi, \xi, \eta, g)$ is said to be locally (globally) D-conformal cosymplectic (l(g).D-c.C.) if the Lee 1-form $\omega$ of $M$ is closed (exact) and

$$
N_{\varphi}=0, \quad \mathrm{~d} \eta=0, \quad \mathrm{~d} \Phi=-2 \Phi \wedge \omega,
$$

where $N_{\varphi}$ is the Nijenhuis tensor of $\varphi$ and $\Phi$ is the fundamental 2-form of the structure $(\varphi, \xi, \eta, g)$.

Remark. Let $(M, \varphi, \xi, \eta, g)$ be an almost contact metric manifold of dimension $2 n+1$ and $L$ the operator given by

$$
L \alpha=\alpha \wedge \Phi
$$

for all $p$-form $\alpha . L$ is an injective homomorphism of the space of $p$-forms into the space of $(p+2)$-forms, for $p \leq n-1$ (see [8]). Thus, is $\operatorname{dim} M \geq 7$ and $\mathrm{d} \Phi=-2 \Phi \wedge \omega$ then $\omega$ is a closed 1-form.

Proposition 4.1. - Let $(M, \varphi, \xi, \eta, g)$ be an almost contact metric manifold. Then $M$ is a l(g). D-c.C. manifold if and only if the Lee 1-form $\omega$ of $M$ is closed (exact) and

$$
\begin{aligned}
\left(\nabla_{X} \varphi\right) Y= & \omega(Y) \varphi X-\omega(\varphi Y) X+\Phi(X, Y) B+ \\
& -g(X, Y) \varphi B+\omega(\varphi Y) \eta(X) \xi+\eta(X) \eta(Y) \varphi B
\end{aligned}
$$

for all $X, Y \in \mathcal{X}(M)$, where $\nabla$ and $B$ are the Riemannian connection of the metric $g$ and the Lee vector field of $M$ respectively.

Proof.- If $(M, \varphi, \xi, \eta, g)$ is an almost contact metric manifold then [2]:

$$
\begin{aligned}
2 g\left(\left(\nabla_{X} \varphi\right) Y, Z\right)= & 3 \mathrm{~d} \Phi(X, \varphi Y, \varphi Z)-3 \mathrm{~d} \Phi(X, Y, Z)+ \\
& +g\left(N^{1}(Y, Z), \varphi X\right)+N^{2}(Y, Z) \eta(X) \\
& +2 \mathrm{~d} \eta(\varphi Y, X) \eta(Z)-2 \mathrm{~d} \eta(\varphi Z, X) \eta(Y),
\end{aligned}
$$

where $N^{1}$ and $N^{2}$ are the tensors defined by

$$
\begin{aligned}
& N^{1}(X, Y)=N_{\varphi}(X, Y)+2 \mathrm{~d} \eta(X, Y) \xi, \\
& N^{2}(X, Y)=\left(\mathcal{L}_{\varphi X} \eta\right) Y-\left(\mathcal{L}_{\varphi} \eta\right) X,
\end{aligned}
$$

being $\mathcal{L}$ the Lie derivative operator. 


\section{Chinea, J. C. Marrero and J. Rocha}

Suppose that $M$ is a $\mathrm{l}(\mathrm{g})$.D-c.C. manifold. Then, from (4.3), (4.6) and (4.7), we obtain (4.5).

Conversely, if $(\varphi, \xi, \eta, g)$ is an almost contact metric structure which satisfies (4.5) then, using (1.1), (1.3), (1.4) and (1.5), we deduce (4.3).

Remark. - If $(M, \varphi, \xi, \eta, g)$ is a l.D-c.C. manifold then, from (4.5), we have that $\nabla_{\xi} \xi=0$. Thus, if $\operatorname{dim} M=3$ and $\omega$ is the Lee 1 -form of $M$, then $\omega=\omega(\xi) \eta$.

Now, we justify definition 4.1.

Proposition 4.2. - Let $(M, \varphi, \xi, \eta, g)$ be an almost contact metric manifold.

(1) If $M$ is a l.D-c.C. manifold then every point $x$ of $M$ has an open neighbourhood $U$ such that the structure $(\varphi, \xi, \eta, \tilde{g})$ is cosymplectic on $U$, where $\sigma: U \rightarrow \mathbb{R}$ is a certain real differentiable function on $U$ and $\tilde{g}=e^{2 \sigma} g+\left(1-e^{2 \sigma}\right) \eta \otimes \eta$. The converse is true if $\operatorname{dim} M \geq 5$.

(2) If $\operatorname{dim} M=3$ and for every point $x$ of $M$ there exists an open neighbourhood $U$ of $x$ and a real differentiable function $\sigma$ on $U$ such that $\mathrm{d} \sigma=\xi(\sigma) \eta$ and the structure $(\varphi, \xi, \eta, \tilde{g})$, with $\tilde{g}=e^{2 \sigma} g+(1-$ $\left.e^{2 \sigma}\right) \eta \otimes \eta$, is cosymplectic on $U$ then $M$ is a l.D-c.C. manifold.

Proof. - Suppose that $(M, \varphi, \xi, \eta, g)$ is a l.D-c.C. manifold. Since the Lee 1-form $\omega$ of $M$ is closed, then for every point $x$ of $M$ there exists an open neighbourhood $U$ of $x$ and a real differentiable function $\sigma$ on $U$ such that $\omega=\mathrm{d} \sigma$ on $U$. Denote by $\Phi$ (respectively $\tilde{\Phi}$ ) the fundamental 2-form of the structure $(\varphi, \xi, \eta, g)$ (respectively $(\varphi, \xi, \eta, \tilde{g})$, with $\left.\tilde{g}=e^{2 \sigma} g+\left(1-2 e^{2 \sigma}\right) \eta \otimes \eta\right)$ on $U$. Then using (4.3) and the fact that

$$
\tilde{\Phi}=e^{2 \sigma} \Phi,
$$

we have that the structure $(\varphi, \xi, \eta, \tilde{g})$ is cosymplectic on $U$.

Conversely, if $\operatorname{dim} M \geq 5$ and every point $x$ of $M$ has an open neighbourhood $U$ such that the structure $(\varphi, \xi, \eta, \tilde{g})$, with $\tilde{g}=e^{2 \sigma} g+\left(1-2 e^{2 \sigma}\right) \eta \otimes \eta$, is cosymplectic on $U$, where $\sigma$ is a real differentiable function on $U$, then we obtain that

$$
N_{\varphi}=0, \quad \mathrm{~d} \eta=0 .
$$

Moreover, from (4.8), we deduce that on each $U$ there is a closed 1-form $\tau_{U}=\mathrm{d} \sigma$ which satisfies $\mathrm{d} \Phi=-2 \tau_{U} \wedge \Phi$. 
Clearly, on each $U \cap U^{\prime}$ we have that $\left(\tau_{U}-\tau_{U^{\prime}}\right) \wedge \Phi=0$. Thus, since the operator $L$ given in (4.4) is injective, we obtain that $\tau_{U}=\tau_{U^{\prime}}$ on $U \cap U^{\prime}$.

Hence, we get. on $M$ a globally defined 1-form $\tau$ verifying

$$
\mathrm{d} \Phi=-2 \tau \wedge \Phi .
$$

Therefore, using (1.1), (1.6), (1.7), (1.8), (4.6), (4.9) and (4.10), we deduce that $\tau$ is the Lee 1 -form of $M$. This proves (1).

The proof of (2) is similar.

\section{5. $D(\sigma)$-conformal cosymplectic submersions}

The results obtained in section 3 suggest us to consider a particular class of almost contact submersions with total space a g.c.C. manifold which are not Riemannian submersions. For these submersions we study the minimality of the fibers, the transference of structures to the base space and to the fibers and the integrability of the horizontal distribution (corollaries 5.1, 5.2 and 5.3).

Let $(M, \varphi, \xi, \eta, g)$ and $\left(M^{\prime}, \varphi^{\prime}, \xi^{\prime}, \eta^{\prime}, g^{\prime}\right)$ be almost contact metric manifolds and $\sigma$ a real differentiable function on $M$.

Definition 5.1. - A smooth surjective mapping $\pi:(M, \varphi, \xi, \eta, g) \rightarrow$ $\left(M^{\prime}, \varphi^{\prime}, \xi^{\prime}, \eta^{\prime}, g^{\prime}\right)$ is called $D(\sigma)$-conformal almost contact submersion if:

(1) $\pi$ is a submersion;

(2) $\pi$ is an almost contact mapping, i.e. $\pi_{*} \circ \varphi=\varphi^{\prime} \circ \pi_{*}$;

(3) for all $x \in M$ and for all $u, v \in T_{x} M$ orthogonals to the vertical space at $x$,

$$
g_{\pi(x)}^{\prime}\left(\pi_{*}^{x} u, \pi_{*}^{x} v\right)=g_{x}(u, v)+\left(e^{2 \sigma(x)}-1\right) \eta_{x}(u) \eta_{x}(v) .
$$

Let $\pi:(M, \varphi, \xi, \eta, g) \rightarrow\left(M^{\prime}, \varphi^{\prime}, \xi^{\prime}, \eta^{\prime}, g^{\prime}\right)$ be a $D(\sigma)$-conformal almost contact submersion. From (2) of definition 5.1, we deduce that the fibers of $\pi$ are invariant submanifolds of $M$. In fact, if $(\tilde{\varphi}, \tilde{\xi}, \tilde{\eta}, \tilde{g})$ is the almost contact metric structure on $M$ given by

$$
\tilde{\varphi}=\varphi, \quad \tilde{\xi}=e^{-\sigma} \xi, \quad \tilde{\eta}=e^{\sigma} \eta, \quad \tilde{g}=g+\left(e^{2 \sigma}-1\right) \eta \otimes \eta,
$$


then $(\tilde{\varphi}, \tilde{\xi}, \tilde{\eta}, \tilde{g})$ is a $D(\sigma)$-conformal almost contact transformation of the structure $(\varphi, \xi, \eta, g)$, and the mapping $\pi$ defines an almost contact metric submersion, which we shall denote by $\tilde{\pi}$, between the almost contact metric manifolds $(M, \tilde{\varphi}, \tilde{\xi}, \tilde{\eta}, \tilde{g})$ and $\left(M^{\prime}, \varphi^{\prime}, \xi^{\prime}, \eta^{\prime}, g^{\prime}\right)$. It is clear that the fibers and the horizontal distribution of $\pi$ coincide with the fibers and the horizontal distribution of $\tilde{\pi}$, respectively. Therefore, if $X$ is a vector field on $M$ then the vertical (respectively horizontal) component of $X$ with respect to $\pi$ coincides with the vertical (respectively horizontal) component of $X$ with respect to $\tilde{\pi}$.

Since $\tilde{\pi}$ is an almost contact metric submersion, we shall suppose that $\tilde{\pi}_{*}(\widetilde{\xi})=\pi_{*}\left(e^{-\sigma} \xi\right)=\xi^{\prime}$. Moreover, from (5.1) and proposition 1.1, we deduce

Proposition 5.1. - Let $\pi:(M, \varphi, \xi, \eta, g) \rightarrow\left(M^{\prime}, \varphi^{\prime}, \xi^{\prime}, \eta^{\prime}, g^{\prime}\right)$ be a $D(\sigma)$-conformal almost contact submersion. Then:

(1) the horizontal and vertical distributions determined by $\pi$ are $\varphi$ invariant. Thus, the fibers of $\pi$ are almost Hermitian manifolds with the induced structure;

(2) for all $X, Y$ and $Z$ basic vector fields on $M$ :

$$
\begin{aligned}
& \pi_{*}\left(N_{\varphi}(X, Y)\right)=N_{\varphi^{\prime}}\left(X_{*}, Y_{*}\right) \circ \pi \\
& \eta^{\prime}\left(X_{*}\right) \circ \pi=e^{\sigma} \eta(X) \\
& \mathrm{d} \eta^{\prime}\left(X_{*}, Y_{*}\right) \circ \pi=e^{\sigma}(\mathrm{d} \sigma \wedge \eta+\mathrm{d} \eta)(X, Y) \\
& \Phi^{\prime}\left(X_{*}, Y_{*}\right) \circ \pi=\Phi(X, Y) \\
& \mathrm{d} \Phi^{\prime}\left(X_{*}, Y_{*}, Z_{*}\right) \circ \pi=\mathrm{d} \Phi(X, Y, Z),
\end{aligned}
$$

where $N_{\varphi}$ (respectively $N_{\varphi^{\prime}}$ ) is the Nijenhuis tensor of $\varphi$ (respectively $\varphi^{\prime}$ ) and $\Phi$ (respectively $\Phi^{\prime}$ ) is the fundamental 2-form of the structure $(\varphi, \xi, \eta, g)$ (respectively $\left(\varphi^{\prime}, \xi^{\prime}, \eta^{\prime}, g^{\prime}\right)$ ).

Now, proposition 3.4 and corollary 3.2 suggest us to introduce a particular class of $D(\sigma)$-conformal almost contact submersions, which we call $D(\sigma)$ conformal cosymplectic submersions.

Definition 5.2.- $A D(\sigma)$-conformal almost contact submersion $\pi$ : $(M, \varphi, \xi, \eta, g) \rightarrow\left(M^{\prime}, \varphi^{\prime}, \xi^{\prime}, \eta^{\prime}, g^{\prime}\right)$ between the almost contact metric manifolds $(M, \varphi, \xi, \eta, g)$ and $\left(M^{\prime}, \varphi^{\prime}, \xi^{\prime}, \eta^{\prime}, g^{\prime}\right)$ is said to be a $D(\sigma)$-conformal cosymplectic submersion if $M$ is a g.c.C. manifold with Lee 1 -form $\omega=\mathrm{d} \sigma$. 
Almost contact submersions with total space a locally conformal cosymplectic manifold

Let $(M, \varphi, \xi, \eta, g)$ be a g.c.C. manifold with Lee 1-form $\omega=\mathrm{d} \sigma$ and $(\tilde{\varphi}, \tilde{\xi}, \tilde{\eta}, \tilde{g})$ the almost contact metric structure on $M$ given by (5.1). Then, it is clear that

$$
N_{\tilde{\varphi}}=0, \quad \mathrm{~d} \tilde{\eta}=0, \quad \mathrm{~d} \tilde{\Phi}=-2 \tilde{\Phi} \wedge \omega,
$$

where $\tilde{\boldsymbol{\Phi}}$ is the fundamental 2 -form of the structure $(\tilde{\varphi}, \tilde{\xi}, \tilde{\eta}, \tilde{g})$. Thus proceeding as in the proof of proposition 4.2 , and if $\operatorname{dim} M \geqq 5$, we obtain that $\omega$ is the Lee 1 -form of $(M, \tilde{\varphi}, \tilde{\xi}, \tilde{\eta}, \tilde{g})$ and therefore $(M, \tilde{\varphi}, \widetilde{\xi}, \tilde{\eta}, \tilde{g})$ is a g.Dc.C. manifold. Analogously, if $\operatorname{dim} M=3$ then we deduce that $\tilde{\omega}=\omega(\xi) \eta$ is the Lee 1 -form of $(M, \tilde{\varphi}, \tilde{\xi}, \tilde{\eta}, \tilde{g})$. Consequently, if the 1-form $\widetilde{\omega}=\omega(\xi) \eta$ is closed we have that $(M, \tilde{\varphi}, \tilde{\xi}, \tilde{\eta}, \tilde{g})$ is also a l.D-c.C. manifold.

Proposition 5.2. - Let $\pi:(M, \varphi, \xi, \eta, g) \rightarrow\left(M^{\prime}, \varphi^{\prime}, \xi^{\prime}, \eta^{\prime}, g^{\prime}\right)$ be a $D(\sigma)$-conformal cosymplectic submersion such that $\omega$ and $\omega^{\prime}$ are the Lee 1-form of $M$ and $M^{\prime}$, respectively.

(1) If $\operatorname{dim} M^{\prime} \geq 5$ and $X$ is a basic vector field then $\omega^{\prime}\left(X_{*}\right) \circ \pi=\omega(X)$.

(2) If $\operatorname{dim} M^{\prime}=3$ and $X$ is a basic vector field then $\omega^{\prime}\left(X_{*}\right) \circ \pi=$ $\omega(\xi) \eta(X)$.

Proof. - Let $(\tilde{\varphi}, \tilde{\xi}, \tilde{\eta}, \tilde{g})$ be the almost contact metric structure on $M$ given by (5.1) and $\tilde{\pi}:(M, \tilde{\varphi}, \tilde{\xi}, \tilde{\eta}, \tilde{g}) \rightarrow\left(M^{\prime}, \varphi^{\prime}, \xi^{\prime}, \eta^{\prime}, g^{\prime}\right)$ the induced almost contact metric submersion by $\pi$. Suppose that $\operatorname{dim} M^{\prime}=2 m^{\prime}+1 \geq 5$. From (1.6), (1.7), (1.8) and proposition 1.1, we obtain that

$$
\begin{aligned}
\omega^{\prime}\left(X_{*}\right) \circ \pi= & \frac{1}{2\left(m^{\prime}-1\right)} \sum_{i=1}^{m^{\prime}}\left(\left(\tilde{\nabla}_{X_{i}} \tilde{\Phi}\right)\left(X_{i}, \tilde{\varphi} X\right)+\left(\tilde{\nabla}_{\tilde{\varphi} X_{i}} \tilde{\Phi}\right)\left(\tilde{\varphi} X_{i}, \tilde{\varphi} X\right)\right)+ \\
& -\frac{1}{2 m^{\prime}} \sum_{i=1}^{m^{\prime}}\left(\left(\tilde{\nabla}_{X_{i}} \tilde{\Phi}\right)\left(\tilde{\xi}, \tilde{\varphi} X_{i}\right)-\left(\tilde{\nabla}_{\tilde{\varphi} X_{i}} \tilde{\Phi}\right)\left(\tilde{\xi}, X_{i}\right)\right) \tilde{\eta}(X),
\end{aligned}
$$

for all $X$ basic vector field on $M$, where $\tilde{\nabla}$ and $\tilde{\Phi}$ are the Riemannian connection of $\tilde{g}$ and the fundamental 2 -form of the structure $(\tilde{\varphi}, \tilde{\xi}, \tilde{\eta}, \tilde{g})$ respectively, and $\left\{X_{1}, \ldots, X_{m^{\prime}}, \tilde{\varphi} X_{1}, \ldots, \tilde{\varphi} X_{m^{\prime}}, \tilde{\xi}\right\}$ is a local orthonormal basis of basic vector fields of the horizontal distribution of $\tilde{\pi}$.

Now, if $\tilde{\omega}$ is the Lee 1 -form of $(M, \tilde{\varphi}, \tilde{\xi}, \tilde{\eta}, \tilde{g})$ then, using (1.1), (4.5) and (5.3), we deduce that $\omega^{\prime}\left(X_{*}\right) \circ \pi=\widetilde{\omega}(X)$. This proves (1).

On the other hand, since $\pi_{*}\left(\tilde{\nabla}_{\tilde{\xi}} \tilde{\xi}\right)=\nabla_{\xi^{\prime}}^{\prime} \xi^{\prime}$ and $\tilde{\nabla} \widetilde{\xi}=0$ we have that $\nabla_{\xi^{\prime}}^{\prime} \xi^{\prime}=0$, being $\nabla^{\prime}$ the Riemannian connection of the metric $g^{\prime}$. Thus, from 
(1.1) we conclude that $\left(\nabla_{\xi^{\prime}}^{\prime} \Phi^{\prime}\right)\left(\xi^{\prime}, X_{*}\right)=0$, where $\Phi^{\prime}$ is the fundamental 2form of the structure $\left(\varphi^{\prime}, \xi^{\prime}, \eta^{\prime}, g^{\prime}\right)$.

Therefore, if $\operatorname{dim} M^{\prime}=3$ and $\left\{X_{1}, \tilde{\varphi} X_{1}, \tilde{\xi}\right\}$ is a local orthonormal basis of basic vector fields of the horizontal distribution of $\tilde{\pi}$ then, using (1.6) and proposition 1.1, we obtain that

$$
\omega^{\prime}\left(X_{*}\right) \circ \pi=-\frac{1}{2}\left(\left(\tilde{\nabla}_{X_{1}} \tilde{\Phi}\right)\left(\tilde{\xi}, \tilde{\varphi} X_{1}\right)-\left(\tilde{\nabla}_{\tilde{\varphi} X_{1}} \tilde{\Phi}\right)\left(\tilde{\xi}, X_{1}\right)\right) \tilde{\eta}(X) .
$$

Finally, from (1.1), (4.5) and (5.4), we deduce that $\omega^{\prime}\left(X_{*}\right) \circ \pi=$ $\omega(\xi) \eta(X)$, which shows $(2)$.

Next, we prove a result which will be useful in the sequel.

Proposition 5.3. - If $\pi:(M, \varphi, \xi, \eta, g) \rightarrow\left(M^{\prime}, \varphi^{\prime}, \xi^{\prime}, \eta^{\prime}, g^{\prime}\right)$ is a $D(\sigma)$ conformal cosymplectic submersion and $X, Y$ horizontal vector fields on $M$, then:

$$
v[X, Y]=2 \Phi(X, Y) \varphi(v B),
$$

where $\boldsymbol{\Phi}$ is the fundamental 2 -form of the structure $(\varphi, \xi, \eta, g)$ and $B$ is the Lee vector field of $M$.

Proof.- If $\operatorname{dim} M=3$ then $\operatorname{dim} M^{\prime}=3$ and $v[X, Y]=v B=0$. Consequently (5.5) is true.

Suppose that $\operatorname{dim} M \geq \mathbf{5}$ and let $(\tilde{\varphi}, \tilde{\xi}, \tilde{\eta}, \tilde{g})$ be the almost contact metric structure on $M$ given in (5.1) and $\tilde{\pi}:(M, \tilde{\varphi}, \tilde{\xi}, \tilde{\eta}, \tilde{g}) \rightarrow\left(M^{\prime}, \varphi^{\prime}, \xi^{\prime}, \eta^{\prime}, g^{\prime}\right)$ the induced almost contact metric submersion by $\pi$. Then, from (5.2), we obtain (4.5) and thus if $X$ and $Y$ are horizontal vector fields we deduce that

$$
\begin{aligned}
\tilde{\nabla}_{X} \varphi Y-\varphi \tilde{\nabla}_{X} Y= & \omega(Y) \varphi X-\omega(\varphi Y) X+\Phi(X, Y) \tilde{B}+ \\
& -\tilde{g}(X, Y) \varphi \widetilde{B}+\omega(\varphi Y) \tilde{\eta}(X) \tilde{\xi}+\tilde{\eta}(X) \tilde{\eta}(Y) \varphi \tilde{B}
\end{aligned}
$$

being $\omega$ and $\Phi$ the Lee 1-form of $M$ and the fundamental 2-form of the structure $(\varphi, \xi, \eta, g)$ respectively and $\tilde{\nabla}$ and $\widetilde{B}$ the Riemannian connection of the metric $\tilde{g}$ and the Lee vector field of $(M, \tilde{\varphi}, \tilde{\xi}, \tilde{\eta}, \tilde{g})$, respectively.

Denote by $\tilde{A}$ the integrability tensor of the submersion $\tilde{\pi}$. Taking vertical components in (5.6) we have that

$$
\tilde{A}_{X} \varphi Y-\varphi \tilde{A}_{X} Y=\Phi(X, Y)(v \widetilde{B})-\tilde{g}(X, Y) \varphi(v \widetilde{B})+\tilde{\eta}(X) \tilde{\eta}(Y) \varphi(v \widetilde{B}) .
$$


Almost contact submersions with total space a locally conformal cosymplectic manifold

Using (5.7) and the fact that $\tilde{A}_{X} Y=-\tilde{A}_{Y} X$ we show that

$$
\tilde{A}_{X} Y+\tilde{A}_{\varphi X} \varphi Y-\tilde{\eta}(X) \tilde{A}_{\tilde{\xi}} Y=2 \Phi(X, Y) \varphi(v \tilde{B}) .
$$

On the other hand, from (4.5), we obtain that

$$
\tilde{A}_{\tilde{\xi}} Y=-\tilde{A}_{Y} \tilde{\xi}=-v\left(\tilde{\nabla}_{Y} \tilde{\xi}\right)=\mathbf{0} .
$$

Next, we shall prove that

$$
\tilde{A}_{\varphi X} \varphi Y=\tilde{A}_{X} Y
$$

Suppose that $X$ is a basic vector field and $V$ a vertical vector field. Then, since $\tilde{A}_{X} V=h\left(\tilde{\nabla}_{V} X\right)$ and $\tilde{g}\left(\tilde{A}_{X} \varphi Y, V\right)=-\tilde{g}\left(\varphi Y, \tilde{A}_{X} V\right)$ (see [19]), we deduce that $\tilde{g}\left(\tilde{A}_{X} \varphi Y, V\right)=\tilde{g}\left(Y, h\left(\varphi\left(\tilde{\nabla}_{V} X\right)\right)\right)$. Now, using (4.5), we have that $h\left(\left(\tilde{\nabla}_{V} \varphi\right) X\right)=0$ and thus $\tilde{g}\left(\tilde{A}_{X} \varphi Y, V\right)=-\tilde{g}\left(\tilde{A}_{\varphi X} Y, V\right)$, which, by (5.9), implies that $\tilde{A}_{\varphi X} \varphi Y=\tilde{A}_{X} Y$.

From (5.8), (5.9) and (5.10), we obtain that $v[X, Y]=2 \Phi(X, Y) \varphi(v \widetilde{B})$, and therefore, using that

$$
\widetilde{B}=B+\left(e^{-2 \sigma}-1\right) \omega(\xi) \xi,
$$

we deduce (5.5).

Let $\pi:(M, \varphi, \xi, \eta, g) \rightarrow\left(M^{\prime}, \varphi^{\prime}, \xi^{\prime}, \eta^{\prime}, g^{\prime}\right)$ be a $D(\sigma)$-conformal cosymplectic submersion. Then the fibers of $\pi$ are l.c.K. manifolds with the induced structure [9, proposition 2.2]. Moreover, if $\operatorname{dim} M^{\prime} \geq 5$ then, from theorem 1.1 and propositions 5.1, 5.2 and 5.3 we have that

$$
N_{\varphi^{\prime}}=0, \quad \mathrm{~d} \eta^{\prime}=0, \quad \mathrm{~d} \Phi^{\prime}=-2 \Phi^{\prime} \wedge \omega^{\prime}, \quad \mathrm{d} \omega^{\prime}=0,
$$

where $\omega^{\prime}$ and $\Phi^{\prime}$ are the Lee 1-form of $M^{\prime}$ and the fundamental 2-form of the structure $\left(\varphi^{\prime}, \xi^{\prime}, \eta^{\prime}, g^{\prime}\right)$, respectively, and $N_{\varphi^{\prime}}$ is the Nijenhuis tensor of $\varphi^{\prime}$. Thus, $\left(M^{\prime}, \varphi^{\prime}, \xi^{\prime}, \eta^{\prime}, g^{\prime}\right)$ is a l.D-c.C. manifold. If $\operatorname{dim} M^{\prime}=3$ and the 1form $\omega(\xi) \eta$ is closed, being $\omega$ the Lee 1-form of $M$, then, using propositions 5.1 and 5.2, we also obtain that $\left(M^{\prime}, \varphi^{\prime}, \xi^{\prime}, \eta^{\prime}, g^{\prime}\right)$ is a l.D-c.C. manifold. Consequently, we conclude 
Corolla RY 5.1. - Let $\pi:(M, \varphi, \xi, \eta, g) \rightarrow\left(M^{\prime}, \varphi^{\prime}, \xi^{\prime}, \eta^{\prime}, g^{\prime}\right)$ be a $D(\sigma)$-conformal cosymplectic submersion and $\omega$ the Lee 1-form of $M$.

(1) The fibers of $\pi$ are l.c.K. manifolds with the induced structure.

(2) If $\operatorname{dim} M^{\prime} \geq 5$ then $\left(M^{\prime}, \varphi^{\prime}, \xi^{\prime}, \eta^{\prime}, g^{\prime}\right)$ is a l.D-c.C. manifold.

(3) If $\operatorname{dim} M^{\prime}=3$ and the 1 -form $\omega(\xi) \eta$ is closed then $\left(M^{\prime}, \varphi^{\prime}, \xi^{\prime}, \eta^{\prime}, g^{\prime}\right)$ is also a l.D-c.C. manifold.

A l.D-c.C. manifold $\left(M^{\prime}, \varphi^{\prime}, \xi^{\prime}, \eta^{\prime}, g^{\prime}\right)$ with Lee 1-form $\omega^{\prime}$ is cosymplectic if and only if $\omega^{\prime}=0$ (definition 4.1).

On the other hand, if $\pi:(M, \varphi, \xi, \eta, g) \rightarrow\left(M^{\prime}, \varphi^{\prime}, \xi^{\prime}, \eta^{\prime}, g^{\prime}\right)$ is a $D(\sigma)$ conformal cosymplectic submersion then, from proposition 2.2 of [9], we obtain that

$$
h\left[\nabla_{U} V+\nabla_{\varphi U} \varphi V\right]=2 g(U, V)(h B)
$$

where $U$ and $V$ are vertical vector fields on $M, \nabla$ is the Riemannian connection of $g$ and $B$ is the Lee vector field of $M$. Thus, we have that the fibers of $\pi$ are minimal submanifolds of $M$ if and only if $B$ is a vertical vector field on $M$ (see also [9, proposition 3.2]).

Using the above facts and proposition 5.2 and corollary 5.1 we deduce

CoROLlaRY 5.2.- Let $\pi:(M, \varphi, \xi, \eta, g) \rightarrow\left(M^{\prime}, \varphi^{\prime}, \xi^{\prime}, \eta^{\prime}, g^{\prime}\right)$ be a $D(\sigma)$-conformal cosymplectic submersion and $\omega$ and $B$ the Lee 1-form and the Lee vector field of $M$, respectively.

(1) The fibers of $\pi$ are minimal submanifolds of $M$ if and only if $B$ is a vertical vector field on $M$.

(2) If $\operatorname{dim} M^{\prime} \geq 5$ then $\left(M^{\prime}, \varphi^{\prime}, \xi^{\prime}, \eta^{\prime}, g^{\prime}\right)$ is a cosymplectic manifold if and only if $B$ is a vertical vector field on $M$.

(3) If $\operatorname{dim} M^{\prime}=3$ then $\left(M^{\prime}, \varphi^{\prime}, \xi^{\prime}, \eta^{\prime}, g^{\prime}\right)$ is a cosymplectic manifold if and only if $\omega(\xi)=0$.

Let $(\bar{M}, \bar{\varphi}, \bar{\xi}, \bar{\eta}, \bar{g})$ be a l.c.C. manifold with Lee vector field $\bar{B}$ and $M$ an invariant submanifold of $\bar{M}$ such that $\bar{\xi}$ is normal to $M$.

If $\bar{B}$ is normal to $M$ then $M$ is a Kähler manifold with the induced structure [9, corollary 2.1]. Conversely, if $M$ is a Kähler manifold with the induced structure and $\operatorname{dim} M \geq 4$ then $\bar{B}$ is normal to $M[9$, proposition 3.1]. Using these facts and proposition 5.3 we obtain the following corollary. 
Almost contact submersions with total space a locally conformal cosymplectic manifold

Corollary 5.3. - Let $\pi:(M, \varphi, \xi, \eta, g) \rightarrow\left(M^{\prime}, \varphi^{\prime}, \xi^{\prime}, \eta^{\prime}, g^{\prime}\right)$ be a $D(\sigma)$-conformal cosymplectic submersion and $B$ the Lee vector field of $M$. Suppose $\operatorname{dim} M=2 m+1$ and $\operatorname{dim} M^{\prime}=2 m^{\prime}+1$. Then:

(1) the vector field $B$ is horizontal on $M$ if and only if the horizontal distribution determined by $\pi$ is completely integrable. In this case the fibers of $\pi$ are Kähler manifolds with the induced structure;

(2) if $m>m^{\prime}+1$ and the fibers of $\pi$ are Kähler manifolds with the induced structure then the horizontal distribution determined by $\pi$ is completely integrable.

\section{6. $D(\sigma)$-conformal cosymplectic submersions with} horizontal Lee vector field and totally umbilical fibers

In this section we obtain the local model of a $D(\sigma)$-conformal cosymplectic submersion with horizontal Lee vector field and totally umbilical fibers (theorem 6.1).

Let $\left(M^{\prime}, \varphi^{\prime}, \xi^{\prime}, \eta^{\prime}, g^{\prime}\right)$ be a g.D-c.C. manifold and $(V, J, h)$ a Kähler manifold. Suppose that $\sigma^{\prime}$ is a real differentiable function on $M^{\prime}$ such that $\mathrm{d} \sigma^{\prime}=\omega^{\prime}$, where $\omega^{\prime}$ is the Lee 1 -form of $M^{\prime}$. On the product manifold $M=M^{\prime} \times V$, we define the almost contact metric structure $(\varphi, \xi, \eta, g)$ given by

$$
\begin{gathered}
\varphi\left(X^{\prime}, X\right)=\left(\varphi^{\prime} X^{\prime}, J X\right), \quad \xi=\left(e^{\sigma^{\prime}} \xi^{\prime}, 0\right), \quad \eta\left(X^{\prime}, X\right)=e^{-\sigma^{\prime}} \eta^{\prime}\left(X^{\prime}\right) \\
g\left(\left(X^{\prime}, X\right),\left(Y^{\prime}, Y\right)\right)=g^{\prime}\left(X^{\prime}, Y^{\prime}\right)+\left(e^{-2 \sigma^{\prime}}-1\right) \eta^{\prime}\left(X^{\prime}\right) \eta^{\prime}\left(Y^{\prime}\right)+ \\
+e^{-2 \sigma^{\prime}} h(X, Y)
\end{gathered}
$$

for all $X, Y$ vector fields on $V$ and $X^{\prime}, Y^{\prime}$ vector fields on $M^{\prime}$.

Let $\tilde{g}^{\prime}$ be the Riemannian metric on $M^{\prime}$ defined by $\tilde{g}^{\prime}=g^{\prime}+\left(e^{-2 \sigma^{\prime}}-\right.$ 1) $\eta^{\prime} \otimes \eta^{\prime}$. Then, the Riemannian manifold $(M, g)$ is the warped product $\left(M^{\prime}, \tilde{g}^{\prime}\right) \times_{e^{-2 \sigma^{\prime}}}(V, h)($ see $[20])$.

We shall denote by $\pi$ the canonical projection of $M$ onto $M^{\prime}$ and by $\left(M^{\prime}, \varphi^{\prime}, \xi^{\prime}, \eta^{\prime}, \tilde{g}^{\prime}\right) \times_{e^{-2 \sigma^{\prime}}}(V, J, h)$ (or simply by $M^{\prime} \times_{e^{-2 \sigma^{\prime}}} V$ ) the manifold $M$ with the almost contact metric structure $(\varphi, \xi, \eta, g)$. We have

Proposition 6.1

(1) The almost contact metric manifold $M=M^{\prime} \times_{e^{-2 \sigma^{\prime}}} V$ is a g.c.C. manifold with Lee 1-form $\pi^{*} \omega^{\prime}$. 
(2) The projection $\pi$ is a $D\left(\sigma^{\prime} \circ \pi\right)$-conformal cosymplectic submersion of $M$ onto $M^{\prime}$ such that the Lee vector field of $M$ is horizontal and the fibers are totally umbilical submanifolds of $M$.

Proof. - Let $N_{\varphi^{\prime}}, N_{J}$ and $N_{\varphi}$ be the Nijenhuis tensors of $\varphi^{\prime}, J$ and $\varphi$, respectively. From (6.1) and by a direct computation we deduce that

$$
\begin{aligned}
& N_{\varphi}\left(\left(X^{\prime}, X\right),\left(Y^{\prime}, Y\right)\right)=\left(N_{\varphi^{\prime}}\left(X^{\prime}, Y^{\prime}\right), N_{J}(X, Y)\right)=0 \\
& \mathrm{~d} \eta=\eta \wedge \pi^{*} \omega^{\prime},
\end{aligned}
$$

for all $X^{\prime}, Y^{\prime}$ vector fields on $M^{\prime}$ and $X, Y$ vector fields on $V$.

Now, if $\Phi$ is the fundamental 2 -form of the structure $(\varphi, \xi, \eta, g)$ then

$$
\Phi=\pi^{*} \Phi^{\prime}+\pi^{*}\left(e^{-2 \sigma^{\prime}}\right) \tau^{*} \Omega
$$

where $\Phi^{\prime}$ and $\Omega$ are the fundamental 2 -form of the structure $\left(\varphi^{\prime}, \xi^{\prime}, \eta^{\prime}, g^{\prime}\right)$ and the Kähler 2-form of the structure $(J, h)$, respectively, and $\tau$ is the canonical projection of $M$ onto $V$.

Thus, using (6.2) and (4.3), we obtain that $\mathrm{d} \Phi=-2\left(\pi^{*} \omega^{\prime}\right) \wedge \Phi$. Therefore $M=M^{\prime} \times{ }_{e^{-2 \sigma^{\prime}}} V$ is a g.c.C. manifold with Lee 1-form $\pi^{*} \omega^{\prime}$.

On the other hand, it is easy to prove that $\pi$ is a $D\left(\sigma^{\prime} \circ \pi\right)$-conformal cosymplectic submersion. Moreover, if $B$ and $B^{\prime}$ are the Lee vector fields of $M$ and $M^{\prime}$, respectively, then, from (6.1) we have that $B=$ $B^{\prime}+\left(e^{2 \sigma^{\prime}}-1\right) \omega^{\prime}\left(\xi^{\prime}\right) \xi^{\prime}$. Consequently $B$ is a horizontal vector field on $M=M^{\prime} \times e_{e^{-2 \sigma^{\prime}}} V$.

Finally, it is clear that the fibers of $\pi$ are $\left\{m^{\prime}\right\} \times V$, with $m^{\prime} \in M^{\prime}$. Thus, by a well-known result of warped products [20], we deduce that $\pi$ is a submersion with totally umbilical fibers.

Remark. - Let $(\bar{\varphi}, \bar{\xi}, \bar{\eta}, \bar{g})$ be the almost contact metric structure on $M=M^{\prime} \times{ }_{e^{-2 \sigma^{\prime}}} V$ given by $\bar{\varphi}=\varphi, \bar{\xi}=e^{-\sigma^{\prime}} \xi, \bar{\eta}=e^{\sigma^{\prime}} \eta, \bar{g}=e^{2 \sigma^{\prime}} g$, and $\bar{g}^{\prime}$ the Riemannian metric on $M^{\prime}$ defined by $\bar{g}^{\prime}=e^{2 \sigma^{\prime}} \tilde{g}^{\prime}$. Then, the almost contact metric manifolds $(M, \bar{\varphi}, \bar{\xi}, \bar{\eta}, \bar{g})$ and $\left(M^{\prime}, \varphi^{\prime}, \xi^{\prime}, \eta^{\prime}, \bar{g}^{\prime}\right)$ are cosymplectic manifolds. In fact, $(M, \bar{\varphi}, \bar{\xi}, \bar{\eta}, \bar{g})$ is the product of the cosymplectic manifold $\left(M^{\prime}, \varphi^{\prime}, \xi^{\prime}, \eta^{\prime}, \bar{g}^{\prime}\right)$ with the Kähler manifold $(V, J, h)$ and $\pi$ is a trivial cosymplectic submersion of $(M, \bar{\varphi}, \bar{\xi}, \bar{\eta}, \bar{g})$ onto $\left(M^{\prime}, \varphi^{\prime}, \xi^{\prime}, \eta^{\prime}, \bar{g}^{\prime}\right)$.

Proposition 6.1 and the above remark suggest us to introduce the following definition. 
Almost contact submersions with total space a locally conformal cosymplectic manifold

Definition 6.1.- $A D(\sigma)$-conformal cosymplectic submersion $\gamma$ : $M \rightarrow N$ is said to be trivial if there exists a g.D-c.C. manifold $M^{\prime}$ and a Kähler manifold $V$ such that the following diagram is commutative

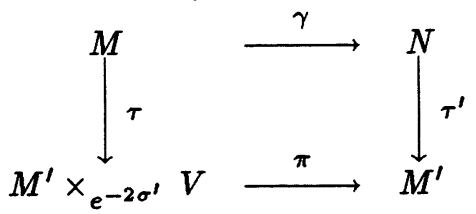

where $\tau$ and $\tau^{\prime}$ are almost contact isometries and $\omega^{\prime}=\mathrm{d} \sigma^{\prime}$ is the Lee 1-form of $M^{\prime}$, being $\sigma^{\prime}$ a real differentiable function on $M^{\prime}$.

From proposition 6.1 we obtain the following corollary.

Corollary 6.1. - If $\gamma: M \rightarrow N$ is a trivial $D(\sigma)$-conformal cosymplectic submersion, then the Lee vector field of $M$ is horizontal and the fibers of $\gamma$ are totally umbilical submanifolds of $M$.

Let $(M, \varphi, \xi, \eta, g)$ be a connected l.c.C. manifold. Then the universal covering space $\bar{M}$ of $M$ has an induced g.c.C. structure $(\bar{\varphi}, \bar{\xi}, \bar{\eta}, \bar{g})$. Thus, there is a real differentiable function $\bar{\sigma}$ on $\bar{M}$ such that $\left(\bar{M}, \bar{\varphi}, e^{-\bar{\sigma}} \bar{\xi}, e^{\bar{\sigma}} \bar{\eta}, e^{2 \bar{\sigma}} \bar{g}\right)$ is a cosymplectic manifold. In fact, if $\bar{\sigma}^{\prime}$ is a real differentiable function on $\bar{M}$ such that $\left(\bar{M}, \bar{\varphi}, e^{-\bar{\sigma}^{\prime}} \bar{\xi}, e^{\bar{\sigma}^{\prime}} \bar{\eta}, e^{2 \bar{\sigma}^{\prime}} \bar{g}\right)$ is a cosymplectic manifold then $\bar{\sigma}^{\prime}=\bar{\sigma}+c$, with $c$ constant ([10] and [11]).

Definition 6.2. - A l.c.C. manifold $(M, \varphi, \xi, \eta, g)$ is said to be locally conformal complete cosymplectic if the Riemannian manifold $\left(\bar{M}, \bar{g}^{\prime}=e^{2 \bar{\sigma}} \bar{g}\right)$ is complete.

Let $\pi:(M, \varphi, \xi, \eta, g) \rightarrow\left(M^{\prime}, \varphi^{\prime}, \xi^{\prime}, \eta^{\prime}, g^{\prime}\right)$ be a $D(\sigma)$-conformal cosymplectic submersion of the connected manifold $M$ onto $M^{\prime}, \bar{M}$ the universal covering space of $M$ and $(\bar{\varphi}, \bar{\xi}, \bar{\eta}, \bar{g})$ the induced g.c.C. structure on $\bar{M}$ by $(\varphi, \xi, \eta, g)$. Then $\pi$ induces a $D(\bar{\sigma})$-conformal cosymplectic submersion of $(M, \bar{\varphi}, \bar{\xi}, \bar{\eta}, \bar{g})$ onto $\left(M^{\prime}, \varphi^{\prime}, \xi^{\prime}, \eta^{\prime}, g^{\prime}\right)$ which we shall denote by $\bar{\pi}$. We shall suppose that the fibers of $\bar{\pi}$ are connected submanifolds of $\bar{M}$.

Now, we shall prove a converse of corollary 6.1.

TheOREM 6.1. - Let $\pi:(M, \varphi, \xi, \eta, g) \rightarrow\left(M^{\prime}, \varphi^{\prime}, \xi^{\prime}, \eta^{\prime}, g^{\prime}\right)$ be a $D(\sigma)$ conformal cosymplectic submersion such that $M$ is a globally conformal complete cosymplectic manifold, the Lee vector field of $M$ is horizontal and the fibers of $\pi$ are totally umbilical submanifolds of $M$. Then, the induced submersion $\bar{\pi}$ of $(\bar{M}, \bar{\varphi}, \bar{\xi}, \bar{\eta}, \bar{g})$ onto $M^{\prime}$ is a trivial $D(\bar{\sigma})$-conformal cosymplectic submersion. 
Proof. - Let $\bar{\omega}$ and $\bar{B}$ be the Lee 1-form and the Lee vector field respectively of $(\bar{M}, \bar{\varphi}, \bar{\xi}, \bar{\eta}, \bar{g})$. Then there is a real differentiable function $\bar{\sigma}$ on $\bar{M}$ such that $\left(\bar{M}, \bar{\varphi}, e^{-\bar{\sigma} \bar{\xi}}, e^{\bar{\sigma}} \bar{\eta}, e^{2 \bar{\sigma}} \bar{g}\right)$ is a cosymplectic manifold and $\bar{\omega}=\mathrm{d} \bar{\sigma}$.

Since $\bar{B}$ is a horizontal vector field then there exists a differentiable function $\sigma^{\prime}$ on $M^{\prime}$ which verifies $\bar{\sigma}=\sigma^{\prime} \circ \bar{\pi}$. Thus, from definition 5.1 and proposition 5.1, we have that $\bar{\pi}$ is a cosymplectic submersion between the cosymplectic manifolds $\left(\bar{M}, \bar{\varphi}, e^{-\bar{\sigma}} \bar{\xi}, e^{\bar{\sigma}} \bar{\eta}, e^{2 \bar{\sigma}} \bar{g}\right)$ and $\left(M^{\prime}, \varphi^{\prime}, \xi^{\prime}, \eta^{\prime}, \bar{g}^{\prime}=\right.$ $\left.e^{2 \sigma^{\prime}} g^{\prime}+\left(1-e^{2 \sigma^{\prime}}\right) \eta^{\prime} \otimes \eta^{\prime}\right)$.

Now, using (5.12), we obtain that the fibers of $\bar{\pi}$ are totally umbilical submanifolds of $(\bar{M}, \bar{g})$ with normal curvature vector field $\bar{B}$. Then, from (2.3), we deduce that the fibers of the submersion $\bar{\pi}$ are totally geodesic submanifolds of $\left(\bar{M}, e^{2 \bar{\sigma}} \bar{g}\right)$. Therefore, since $\left(\bar{M}, e^{2 \bar{\sigma}} \bar{g}\right)$ is a complete Riemannian manifold, we have that $\bar{\pi}$ is a trivial cosymplectic submersion which implies that $\left(\bar{M}, \bar{\varphi}, e^{-\bar{\sigma}} \bar{\xi}, e^{\bar{\sigma}} \bar{\eta}, e^{2 \bar{\sigma}} \bar{g}\right)$ is the product of a Kähler manifold $(V, J, h)$ with the cosymplectic manifold $\left(M^{\prime}, \varphi^{\prime}, \xi^{\prime}, \eta^{\prime}, \bar{g}^{\prime}\right)$ and that $\bar{\pi}$ is the natural projection of $\bar{M}$ onto $M^{\prime}$ (see [6]).

Consequently, the g.c.C. manifold $(\bar{M}, \bar{\varphi}, \bar{\xi}, \bar{\eta}, \bar{g})$ is the product $M^{\prime} \times e^{-2 \sigma^{\prime}} V$.

This ends the proof of theorem.

\section{7. $D(\sigma)$-conformal cosymplectic submersions with total space a PC-manifold}

In this section, we shall study $D(\sigma)$-conformal cosymplectic submersions with total space a PC-manifold. For this purpose, we prove the following result.

LeMma 7.1. - Let $\pi:(M, \varphi, \xi, \eta, g) \rightarrow\left(M^{\prime}, \varphi^{\prime}, \xi^{\prime}, \eta^{\prime}, g^{\prime}\right)$ be a $D(\sigma)$ conformal cosymplectic submersion, $B$ and $B^{\prime}$ the Lee vector fields of $M$ and $M^{\prime}$, respectively, and $\omega$ the Lee 1-form of $M$. If $\operatorname{dim} M^{\prime} \geq 5$ and $\omega(\xi)=0$ then $h B$ is a basic vector field and $(h B)_{*}=B^{\prime}$.

Proof.- Denote by $(\tilde{\varphi}, \tilde{\xi}, \tilde{\eta}, \tilde{g})$ the almost contact metric structure on $M$ given by $(5.1)$ and by $\tilde{\pi}:(M, \tilde{\varphi}, \tilde{\xi}, \tilde{\eta}, \tilde{g}) \rightarrow\left(M^{\prime}, \varphi^{\prime}, \xi^{\prime}, \eta^{\prime}, g^{\prime}\right)$ the almost contact metric submersion induced by $\pi$.

The 1-form $\omega$ is the Lee 1-form of $(M, \tilde{\varphi}, \tilde{\xi}, \tilde{\eta}, \tilde{g})$. Moreover, using (5.11), we deduce that $B$ is the Lee vector field of $(M, \tilde{\varphi}, \tilde{\xi}, \tilde{\eta}, \tilde{g})$. 
On the other hand, if $C$ is the basic vector field associated to $B^{\prime}$ then, from proposition 5.2 , we obtain that $\tilde{g}(C, X)=\tilde{g}(h B, X)$, for all basic vector field $X$ on $M$. Consequently, $C=h B$.

Next, we show that the base space of a $D(\sigma)$-conformal cosymplectic submersion with total space a PC-manifold is a l.D-c.C. manifold with parallel Lee form or a cosymplectic manifold.

Proposition 7.1. - Let $\pi:(M, \varphi, \xi, \eta, g) \rightarrow\left(M^{\prime}, \varphi^{\prime}, \xi^{\prime}, \eta^{\prime}, g^{\prime}\right)$ be a $D(\sigma)$-conformal cosymplectic submersion such that $M$ is a PC-manifold.

(1) If $\operatorname{dim} M^{\prime} \geq 5$ then $\left(M^{\prime}, \varphi^{\prime}, \xi^{\prime}, \eta^{\prime}, g^{\prime}\right)$ is a l.D-c.C. manifold with parallel Lee form.

(2) If $\operatorname{dim} M^{\prime}=3$ then $\left(M^{\prime}, \varphi^{\prime}, \xi^{\prime}, \eta^{\prime}, g^{\prime}\right)$ is a cosymplectic manifold.

Proof. - Suppose that $\operatorname{dim} M^{\prime} \geq 5$. Let $(\tilde{\varphi}, \tilde{\xi}, \tilde{\eta}, \tilde{g})$ be the almost contact metric structure on $M$ given by $(5.1)$ and $\tilde{\pi}:(M, \tilde{\varphi}, \tilde{\xi}, \tilde{\eta}, \tilde{g}) \rightarrow$ $\left(M^{\prime}, \varphi^{\prime}, \xi^{\prime}, \eta^{\prime}, g^{\prime}\right)$ the almost contact metric submersion induced by $\pi$. Denote by $\omega$ and $B$ the Lee 1 -form and the Lee vector field of $M$, respectively. Since $\omega(\xi)=0$, we have that $B$ is the Lee vector field of $(M, \tilde{\varphi}, \tilde{\xi}, \tilde{\eta}, \tilde{g})$ (see (5.11)).

Now, using (5.1), theorem 1.1, the Koszul formula [15, vol. I, p. 160] and the fact that $\omega(\xi)=0$, we deduce that

$$
\tilde{g}\left(\tilde{\nabla}_{X} B, Y\right)=g\left(\nabla_{X} B, Y\right)+c^{2} \eta(X) \eta(Y),
$$

for all $X, Y \in \mathcal{X}(M)$, where $\nabla$ and $\tilde{\nabla}$ are the Riemannian connections of the metrics $g$ and $\tilde{g}$, respectively and $c=\|\omega\|$. Thus, from (1.11) and (7.1), we obtain that $\tilde{\nabla} B=0$. Therefore, if $X$ is a basic vector field of $M$ then

$$
\tilde{\nabla}_{X}(h B)=-\tilde{\nabla}_{X}(v B) .
$$

From (7.2), we have that

$$
h\left(\tilde{\nabla}_{X}(h B)\right)=-\tilde{A}_{X}(v B)
$$

being $\tilde{A}$ the integrability tensor of the submersion $\tilde{\pi}$. 
On the other hand, if $Y$ is a horizontal vector field then, using (5.5) and since

$$
\tilde{g}\left(\tilde{A}_{X} v B, Y\right)=-\tilde{g}\left(v B, \tilde{A}_{X} Y\right)=-\frac{1}{2} \tilde{g}(v B, v[X, Y]),
$$

we prove that $\tilde{g}\left(\tilde{A}_{X} v B, Y\right)=0$. This implies that $\tilde{A}_{X} v B=0$ and thus, by (7.3), we deduce that

$$
h\left(\tilde{\nabla}_{X}(h B)\right)=0 .
$$

Let $B^{\prime}$ be the Lee vector field of $M^{\prime}$ and $\nabla^{\prime}$ the Riemannian connection of the metric $g^{\prime}$. Since $\tilde{\pi}$ is a Riemannian submersion, we have that $\tilde{\pi}_{*}\left(h\left(\tilde{\nabla}_{Y} Z\right)\right)=\nabla_{Y_{*}}^{\prime} Z_{*}$, for all basic vector fields $Y, Z$ on $M$ (see [19]). Therefore, from (7.4) and lemma 7.1, we conclude that $\nabla_{X_{*}}^{\prime} B^{\prime}=0$.

Consequently the vector field $B^{\prime}$ is parallel which shows that the Lee 1-form of $M^{\prime}$ is parallel.

Finally, if $\operatorname{dim} M^{\prime}=3$ then, using corollary 5.2 , we obtain that $M^{\prime}$ is a cosymplectic manifold.

We also deduce

Proposition 7.2. - Let $\pi:(M, \varphi, \xi, \eta, g) \rightarrow\left(M^{\prime}, \varphi^{\prime}, \xi^{\prime}, \eta^{\prime}, g^{\prime}\right)$ be a $D(\sigma)$-conformal cosymplectic submersion such that $M$ is a PC-manifold and $\operatorname{dim} M>\operatorname{dim} M^{\prime}$. Then the Lee vector field of $M$ can not be a horizontal vector field.

Proof. - Let $\omega$ and $B$ the Lee 1-form and the Lee vector field of $M$, respectively, and $\Phi$ the fundamental 2 -form of the structure $(\varphi, \xi, \eta, g)$. Then, we shall use the following notation

$$
c=\|\omega\|, \quad u=\frac{\omega}{c}, \quad U=\frac{B}{c}, \quad v=-u \circ \varphi, \quad V=\varphi U .
$$

Suppose that $U$ is a horizontal vector field. We shall prove that $\Phi\left(W, W^{\prime}\right)=0$, for all vertical vector fields $W$ and $W^{\prime}$, which is a contradiction since $\operatorname{dim} M>\operatorname{dim} M^{\prime}$.

In fact, if $U$ is a horizontal vector field then it is clear that

$$
u(W)=u\left(W^{\prime}\right)=v(W)=v\left(W^{\prime}\right)=0 .
$$

Thus, from (1.12) and (7.5), we have that

$$
\Phi\left(W, W^{\prime}\right)=-\frac{1}{c} \mathrm{~d} v\left(W, W^{\prime}\right) .
$$


Almost contact submersions with total space a locally conformal cosymplectic manifold

Now, using that the vector field $\left[W, W^{\prime}\right]$ is vertical, we deduce that

$$
2 \mathrm{~d} v\left(W, W^{\prime}\right)=W\left(v\left(W^{\prime}\right)\right)-W^{\prime}(v(W))-v\left[W, W^{\prime}\right]=0 .
$$

Consequently, by (7.6), we obtain that $\Phi\left(W, W^{\prime}\right)=0$.

Finally, from corollary 5.3 and proposition 7.2 , we have that

Corollary 7.1. - Let $\pi:(M, \varphi, \xi, \eta, g) \rightarrow\left(M^{\prime}, \varphi^{\prime}, \xi^{\prime}, \eta^{\prime}, g^{\prime}\right)$ be a $D(\sigma)$-conformal cosymplectic submersion being $(M, \varphi, \xi, \eta, g)$ a PC-manifold and $\operatorname{dim} M>\operatorname{dim} M^{\prime}$. Then the horizontal distribution determined by $\pi$ is not completely integrable. Moreover, if $\operatorname{dim} M=2 m+1, \operatorname{dim} M^{\prime}=2 m^{\prime}+1$ and $m>m^{\prime}+1$ then the fibers of $\pi$, with the induced structure, are not Kähler manifolds.

\section{8. $D(\sigma)$-conformal cosymplectic submersions with} total space a PC-manifold and vertical Lee vector field

Firstly we recall and prove some results on cosymplectic manifolds and cosymplectic submersions.

If $\pi:(M, \varphi, \xi, \eta, g) \rightarrow\left(M^{\prime}, \varphi^{\prime}, \xi^{\prime}, \eta^{\prime}, g^{\prime}\right)$ is an almost contact metric submersion we shall denote by $K$ the $\varphi$-sectional curvature of $M$ and by $H$ the holomorphic sectional curvature of the fibers.

An almost contact metric manifold $(M, \varphi, \xi, \eta, g)$ is said to be quasi- $K$ cosymplectic (or quasi-cosymplectic) if ([4] and [21]):

$$
\left(\nabla_{X} \varphi\right) Y+\left(\nabla_{\varphi X} \varphi\right) \varphi Y=\eta(Y) \nabla_{\varphi X} \xi .
$$

It is clear that a cosymplectic manifold is quasi $K$-cosymplectic. In [6], D. Chinea shows the following proposition.

Proposition 8.1. - Let $\pi:(M, \varphi, \xi, \eta, g) \rightarrow\left(M^{\prime}, \varphi^{\prime}, \xi^{\prime}, \eta^{\prime}, g^{\prime}\right)$ be an almost contact metric submersion such that $(M, \varphi, \xi, \eta, g)$ is a quasi- $K$ cosymplectic manifold. Then:

(1) $K(V) \geq H(V)$, for all vertical vector $V$, and equality holds if and only if the fibers of $\pi$ are totally geodesic submanifolds of $M$.

(2) If $(M, \varphi, \xi, \eta, g)$ is a quasi-K-cosymplectic manifold of constant $\varphi$-sectional curvature $k$ then $\left(M^{\prime}, \varphi^{\prime}, \xi^{\prime}, \eta^{\prime}, g^{\prime}\right)$ is also a quasi-Kcosymplectic manifold of constant $\varphi^{\prime}$-sectional curvature $k$. 
Let $(V, J, h)$ be a Kähler manifold of constant holomorphic sectional curvature $k$ and $M$ the product manifold $V \times \mathbb{R}$. Consider on $M$ the almost contact metric structure $(\varphi, \xi, \eta, g)$ given by (3.1). Then $(M, \varphi, \xi, \eta, g)$ is a cosymplectic manifold of constant $\varphi$-sectional curvature $k$. In fact, in [13], S. Eum proves

Proposition 8.2. - Let $(M, \varphi, \xi, \eta, g)$ be a $(2 m+1)$-dimensional complete simply connected cosymplectic manifold of constant $\varphi$-sectional curvature $k$.

(1) If $k>0$ then $M$ is almost contact isometric to $P_{m}\left(C^{m+1}\right)(k) \times \mathbb{R}$.

(2) If $k=0$ then $M$ is almost contact isometric to $C^{m}(0) \times \mathbb{R}$.

(3) If $k<0$ then $M$ is almost contact isometric to $C D^{m}(k) \times \mathbb{R}$.

Now, we introduce the following definition.

DEFINITION 8.1. - Two mappings

$$
\pi_{1}:\left(M_{1}, \varphi_{1}, \xi_{1}, \eta_{1}, g_{1}\right) \rightarrow\left(M_{1}^{\prime}, \varphi_{1}^{\prime}, \xi_{1}^{\prime}, \eta_{1}^{\prime}, g_{1}^{\prime}\right)
$$

and

$$
\pi_{2}:\left(M_{2}, \varphi_{2}, \xi_{2}, \eta_{2}, g_{2}\right) \rightarrow\left(M_{2}^{\prime}, \varphi_{2}^{\prime}, \xi_{2}^{\prime}, \eta_{2}^{\prime}, g_{2}^{\prime}\right)
$$

between almost contact metric manifolds are equivalent if the following diagram is commutative:

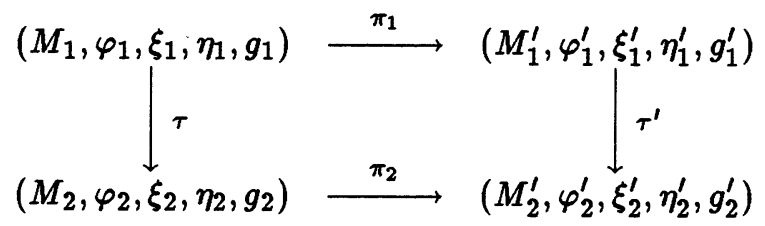

where $\tau$ and $\tau^{\prime}$ are almost contact isometries.

We obtain the following result.

Proposition 8.3. - Let $\pi:(M, \varphi, \xi, \eta, g) \rightarrow\left(M^{\prime}, \varphi^{\prime}, \xi^{\prime}, \eta^{\prime}, g^{\prime}\right)$ be a cosymplectic submersion with connected and totally geodesic fibers and such that $M$ is a complete simply connected cosymplectic manifold of constant $\varphi$ sectional curvature $k$. Suppose that $\operatorname{dim} M=2 m+1$ and $\operatorname{dim} M^{\prime}=2 m^{\prime}+1$, $\boldsymbol{m} \geq \boldsymbol{m}^{\prime}$.

(1) If $k \neq 0$ then $\pi$ is almost contact isometry.

(2) If $k=0$ then $\pi$ and the natural projection of $C^{m}(0) \times \mathbb{R}$ onto $C^{m^{\prime}}(0) \times \mathbb{R}$ are equivalent. 
Proof. - We have that $(M, \varphi, \xi, \eta, g)$ is the product of a complete simply connected Kähler manifold $\left(M_{1}, J_{1}, g_{1}\right)$ with a complete simply connected cosymplectic manifold $\left(M_{1}^{\prime}, \varphi_{1}^{\prime}, \xi_{1}^{\prime}, \eta_{1}^{\prime}, g_{1}^{\prime}\right)$ and that $\pi$ is equivalent to a projection,

$$
\pi: M \simeq M_{1}^{\prime} \times M_{1} \rightarrow M_{1}^{\prime} \simeq M^{\prime} .
$$

Moreover, using proposition 8.1 , we deduce that $\left(M_{1}, J_{1}, g_{1}\right)$ is a Kähler manifold of constant holomorphic sectional curvature $k$ and that $\left(M_{1}^{\prime}, \varphi_{1}^{\prime}, \xi_{1}^{\prime}, \eta_{1}^{\prime}, g_{1}^{\prime}\right)$ is a cosymplectic manifold of constant $\varphi_{1}^{\prime}$-sectional curvature $k$.

If $k=0$ then there exists an almost complex isometry $\tau_{1}$ of $M_{1}$ onto $C^{m-m^{\prime}}(0)$ and an almost contact isometry $\tau^{\prime}$ of $M_{1}^{\prime}$ onto $C^{m^{\prime}}(0) \times \mathbb{R}$ (proposition 8.2 and [15, vol. II, p. 170]) such that the following diagram is commutative

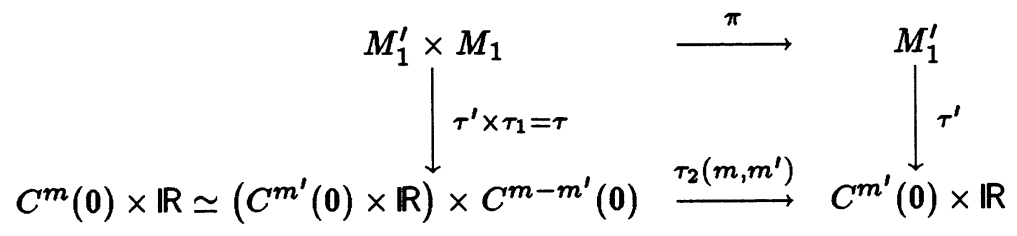

where $\tau_{2}\left(m, m^{\prime}\right)$ is the projection of $C^{m}(0) \times \mathbb{R}$ onto $C^{m^{\prime}}(0) \times \mathbb{R}$. This proves (2).

Now, suppose that $k \neq 0$ and $\operatorname{dim}\left(M_{1}\right) \geq 2$. Then, if $x_{1} \in M_{1}$ and $x_{1}^{\prime} \in M_{1}^{\prime}$ we obtain, by a straightforward computation, that

$$
K_{\left(x_{1}^{\prime}, x_{1}\right)}\left(u_{1}^{\prime}, u_{1}\right)=\frac{1}{4}\left(\left(K_{1}^{\prime}\right)_{x_{1}^{\prime}} u_{1}^{\prime}+\left(H_{1}\right)_{x_{1}} u_{1}\right)=\frac{k}{2}
$$

being $u_{1}$ and $u_{1}^{\prime}$ unit tangent vectors to $M_{1}$ and $M_{1}^{\prime}$ at $x_{1}$ and $x_{1}^{\prime}$ respectively, $H_{1}$ the holomorphic sectional curvature of $M_{1}$ and $K$ (respectively $K_{1}^{\prime}$ ) the $\varphi$-sectional (respectively $\varphi_{1}^{\prime}$-sectional) curvature of $M$ (respectively $\left.M_{1}^{\prime}\right)$.

Using (8.1) we deduce that $k=k / 2$ which is a contradiction since $k \neq 0$.

Therefore, $\operatorname{dim} M_{1}=0$ and thus $\pi$ is an almost contact isometry.

Next, we shall study $D(\sigma)$-conformal cosymplectic submersions with total space a PC-manifold and vertical Lee vector field.

Let $(\bar{M}, \bar{\varphi}, \bar{\xi}, \bar{\eta}, \bar{g})$ be a l.c.C. manifold with Lee vector field $\bar{B}$ and Lee 1-form $\bar{\omega}$ and $M$ an invariant submanifold of $\bar{M}$. Then the mean curvature 
vector field of $M$ is $\bar{B}^{N}$, where $\bar{B}^{N}$ is the normal component to $M$ of $\bar{B}$ [9, proposition 3.2]. Consequently the vector field $\bar{B}$ is tangent to $M$ if and only if $M$ is a minimal submanifold of $\bar{M}$.

If the vector field $\bar{\xi}$ is normal to $M$ and $(\varphi, g)$ is the induced almost Hermitian structure on $M$, then $(M, \varphi, g)$ is a l.c.K. manifold with Lee 1 -form $-2 \omega$, being $\omega$ the 1 -form induced by $\bar{\omega}$ on $M$ [9, proposition 2.2].

Moreover, we have

Proposition 8.4. - Let $(\bar{M}, \bar{\varphi}, \bar{\xi}, \bar{\eta}, \bar{g})$ be a PC-manifold and $M$ an invariant submanifold of $\bar{M}$ such that the vector field $\bar{\xi}$ is normal to $M$. Then, are equivalent:

(1) $M$ is a l.c.K. manifold with parallel Lee form;

(2) the Lee vector field of $\bar{M}$ is tangent to $M$;

(3) $M$ is a minimal submanifold of $\bar{M}$.

Proof. - Denote by $B$ the tangent component to $M$ of $\bar{B}$. Then $-2 B$ is the Lee vector field of the l.c.K. manifold $(M, \varphi, g)$.

Suppose that $(M, \varphi, g)$ is a l.c.K. manifold with parallel Lee 1-form. Using (1.11) and the Gauss formula we obtain that $\bar{\nabla}_{X} \bar{B}^{N}=-\alpha(X, B)$, for any $X \in \mathcal{X}(M)$, where $\bar{\nabla}$ is the Riemannian connection of the metric $\bar{g}$ and $\alpha$ is the second fundamental form of $M$.

Thus, if $X$ and $Y$ are vector fields on $M$ then, since $\bar{g}\left(\alpha(X, Y), \bar{B}^{N}\right)=$ $-\bar{g}\left(\bar{\nabla}_{X} \bar{B}^{N}, Y\right)$, we deduce that $\alpha(X, Y)$ is normal to $\bar{B}^{N}$. As $\bar{B}$ is the mean curvature vector field of $M$, we conclude that $\bar{B}^{N}=0$.

Conversely, if the vector field $\bar{B}$ is tangent to $M$ then, from (1.11) and using the Gauss formula, we have that $\nabla_{X} B=0$, for any $X \in \mathcal{X}(M)$, being $\nabla$ the Riemannian connection of the metric $g$ on $M$.

Therefore, the 1-form $\omega$ on $M$ is parallel and $(M, \varphi, g)$ is a l.c.K. manifold with parallel Lee form.

From proposition 8.4 and corollary 5.2 we obtain

Corollary 8.1. - Let $\pi:(M, \varphi, \xi, \eta, g) \rightarrow\left(M^{\prime}, \varphi^{\prime}, \xi^{\prime}, \eta^{\prime}, g^{\prime}\right)$ be a $D(\sigma)$-conformal cosymplectic submersion with $M$ a PC-manifold.

(a) If $\operatorname{dim} M^{\prime} \geq 5$, then are equivalent:

(1) the fibers of $\pi$, with the induced structure, are l.c.K. manifolds with parallel Lee form; 
(2) the fibers of $\pi$ are minimal submanifolds of $M$;

(3) $\left(M^{\prime}, \varphi^{\prime}, \xi^{\prime}, \eta^{\prime}, g^{\prime}\right)$ is a cosymplectic manifold;

(4) the Lee vector field of $M$ is vertical.

(b) If $\operatorname{dim} M^{\prime}=3$ then $\left(M^{\prime}, \varphi^{\prime}, \xi^{\prime}, \eta^{\prime}, g^{\prime}\right)$ is a cosymplectic manifold and (1), (2) and (4) are equivalent.

Let $(M, \varphi, \xi, \eta, g)$ be a $\mathrm{PC}(k)$-manifold with Lee 1 -form $\omega=\mathrm{d} \sigma, \operatorname{dim} M \geq$ 5, $x \in M$ and $j: F \rightarrow M$ the immersion of the leaf $F$ over $x$ of the canonical foliation $\mathcal{F}$ on $M$.

Consider on $F$ the almost contact metric structure $\left(\varphi_{F}, \xi_{F}, \eta_{F}, g_{F}\right)$ given by (1.13). $\left(F, \varphi_{F}, \xi_{F}, \eta_{F}, g_{F}\right)$ is a $c$-Sasakian manifold of constant $\varphi_{F^{-}}$ sectional curvature $k$, being $c=\|\omega\|(\S 1.4)$.

On the other hand, denote by $(\tilde{\varphi}, \tilde{\xi}, \tilde{\eta}, \tilde{g})$ the almost contact metric structure on $M$ given by (5.1) and by $\tilde{\mathcal{F}}$ the foliation defined by $\tilde{\eta}=0$, $\omega=0$. Then the 1 -form $\omega$ is the Lee 1 -form of $(M, \tilde{\varphi}, \tilde{\xi}, \tilde{\eta}, \tilde{g}),(M, \tilde{\varphi}, \tilde{\xi}, \tilde{\eta}, \tilde{g})$ is a g.D-c.C. manifold with parallel Lee form $(\tilde{\nabla} B=0)$ and it is clear that the foliation $\tilde{\mathcal{F}}$ coincides with the foliation $\mathcal{F}$.

Since $\omega(\tilde{\xi})=0$ then, if $\tilde{\nabla}$ is the Riemannian connection of the metric $\tilde{g}$, from $(1.1),(1.2)$ and $(4.5)$, we deduce that $\tilde{\nabla} \tilde{\eta}=0$. Thus, the foliation $\mathcal{F}$ on the Riemannian manifold $(M, \tilde{g})$ is totally geodesic. Moreover,

$$
g_{F}=j^{*} g=j^{*} \tilde{g}
$$

Now, if $v$ is a tangent unit vector to $M$ at $x$ such that $\eta_{x}(v)=\omega_{x}(v)=$ $\left(\omega_{x} \circ \varphi_{x}\right)(v)=0$ then, $v$ is tangent to $F$ at $x$ and, from (1.13), we obtain that $v$ is normal to $\left(\xi_{F}\right)_{x}$ and $\left(\varphi_{F}\right)_{x} v=\varphi_{x} v=\tilde{\varphi}_{x} v$. Therefore, using (8.2) and the fact that the foliation $\mathcal{F}$ on the Riemannian manifolds $(M, g)$ and $(M, \tilde{g})$ is totally geodesic [16], we have

$$
k=\left(K_{F}\right)_{x} v=K_{x} v=\tilde{K}_{x} v
$$

where $K_{F}$ is the $\varphi_{F}$-sectional curvature on $F$ and $K$ (respectively $\tilde{K}$ ) is the $\varphi$-sectional (respectively $\tilde{\varphi}$-sectional) curvature of $(M, \varphi, \xi, \eta, g)$ (respectively $(M, \tilde{\varphi}, \tilde{\xi}, \tilde{\eta}, \tilde{g}))$.

Next, using (8.3), we shall prove that the base space of a $D(\sigma)$-conformal cosymplectic submersion $\pi:(M, \varphi, \xi, \eta, g) \rightarrow\left(M^{\prime}, \varphi^{\prime}, \xi^{\prime}, \eta^{\prime}, g^{\prime}\right)$ with total space a $\mathrm{PC}(k)$-manifold and vertical Lee vector field is a cosymplectic manifold of constant $\varphi^{\prime}$-sectional curvature. 
Proposition 8.5. - Let $\pi:(M, \varphi, \xi, \eta, g) \rightarrow\left(M^{\prime}, \varphi^{\prime}, \xi^{\prime}, \eta^{\prime}, g^{\prime}\right)$ be a $D(\sigma)$-conformal cosymplectic submersion of a $\mathrm{PC}(k)$-manifold $M$ onto $M^{\prime}$ such that the Lee vector field of $(M, \varphi, \xi, \eta, g)$ is vertical. Then $\left(M^{\prime}, \varphi^{\prime}, \xi^{\prime}, \eta^{\prime}, g^{\prime}\right)$ is a cosymplectic manifold of constant $\varphi^{\prime}$-sectional curvature $k+3 c^{2}$, being $c=\|\omega\|$ and $\omega$ the Lee 1 -form of $(M, \varphi, \xi, \eta, g)$.

Proof.- Denote by $K^{\prime}$ the $\varphi^{\prime}$-sectional curvature of $M^{\prime}$, by $\tilde{\pi}$ : $(M, \tilde{\varphi}, \tilde{\xi}, \tilde{\eta}, \tilde{g}) \rightarrow\left(M^{\prime}, \varphi^{\prime}, \xi^{\prime}, \eta^{\prime}, g^{\prime}\right)$ the almost contact metric submersion induced by $\pi$ and by $B$ the Lee vector field of $(M, \varphi, \xi, \eta, g)$.

Let $x^{\prime}$ be a point of $M^{\prime}$ and $v^{\prime}$ a tangent unit vector to $M^{\prime}$ at $x^{\prime}$, which is normal to $\xi_{x^{\prime}}^{\prime}$.

From theorem 4.5 of [6], we obtain that

$$
\tilde{K}_{x}(v)=K_{x^{\prime}}^{\prime}\left(v^{\prime}\right)-3\left\|\left(\tilde{A}_{x}\right)_{v} \varphi_{x} v\right\|^{2},
$$

where $\tilde{A}$ is the integrability tensor of $\tilde{\pi}$ and $v$ is a horizontal vector at $x \in M$ such that

$$
\pi(x)=x^{\prime}, \quad \pi_{*}^{x}(v)=v^{\prime} .
$$

It is clear that $\eta_{x}(v)=0$. Then, using (5.5) and the fact that $B$ is vertical, we deduce that

$$
\left\|\left(\tilde{A}_{x}\right)_{v} \varphi_{x} v\right\|^{2}=c^{2} .
$$

On the other hand, since $v$ is a horizontal vector then it is normal to $B_{x}$ and $\varphi_{x} B_{x}$. Thus, from (8.3), (8.4) and (8.5), we have that

$$
K_{x^{\prime}}^{\prime} v^{\prime}=k+3 c^{2} \text {. }
$$

Now, we shall prove a result which will be useful is the sequel.

Let

$$
\pi:(M, \varphi, \xi, \eta, g) \rightarrow\left(M^{\prime}, \varphi^{\prime}, \xi^{\prime}, \eta^{\prime}, g^{\prime}\right)
$$

and

$$
\tau:\left(M^{\prime}, \varphi^{\prime}, \xi^{\prime}, \eta^{\prime}, g^{\prime}\right) \rightarrow\left(M^{\prime \prime}, \varphi^{\prime \prime}, \xi^{\prime \prime}, \eta^{\prime \prime}, g^{\prime \prime}\right)
$$

be almost contact metric submersions such that the fibers of $\tau \circ \pi$ are totally geodesic submanifolds of $(M, g)$. Then the fibers of $\tau$ are totally geodesic submanifolds of $\left(M^{\prime}, g^{\prime}\right)$ (see [17]).

We shall show that this result is also true if $\pi$ is a $D(\sigma)$-conformal cosymplectic submersion. 
Almost contact submersions with total space a locally conformal cosymplectic manifold

LEMMA 8.1. - Let

$$
\pi:(M, \varphi, \xi, \eta, g) \rightarrow\left(M^{\prime}, \varphi^{\prime}, \xi^{\prime}, \eta^{\prime}, g^{\prime}\right)
$$

be a $D(\sigma)$-conformal cosymplectic submersion and

$$
\tau:\left(M^{\prime}, \varphi^{\prime}, \xi^{\prime}, \eta^{\prime}, g^{\prime}\right) \rightarrow\left(M^{\prime \prime}, \varphi^{\prime \prime}, \xi^{\prime \prime}, \eta^{\prime \prime}, g^{\prime \prime}\right)
$$

an almost contact metric submersion such that the fibers of $\tau \circ \pi$ are totally geodesic submanifolds of $(M, g)$. Then the fibers of $\tau$ are totally geodesic submanifolds of $\left(M^{\prime}, g^{\prime}\right)$.

Proof.- A straightforward computation shows that the mapping $\tau \circ \pi$ is a $D(\sigma)$-conformal cosymplectic submersion.

Denote by $(\tilde{\varphi}, \tilde{\xi}, \tilde{\eta}, \tilde{g})$ the almost contact metric structure on $M$ given by $(5.1)$ and by $\tilde{\pi}:(M, \tilde{\varphi}, \tilde{\xi}, \tilde{\eta}, \tilde{g}) \rightarrow\left(M^{\prime}, \varphi^{\prime}, \xi^{\prime}, \eta^{\prime}, g^{\prime}\right)$ (respectively $\widetilde{\tau \circ \pi}:$ $\left.(M, \tilde{\varphi}, \tilde{\xi}, \tilde{\eta}, \tilde{g}) \rightarrow\left(M^{\prime \prime}, \varphi^{\prime \prime}, \xi^{\prime \prime}, \eta^{\prime \prime}, g^{\prime \prime}\right)\right)$ the almost contact metric submersion induced by $\pi$ (respectively $\tau \circ \pi$ ).

It is clear that $\widetilde{\tau \circ \pi}=\tau \circ \tilde{\pi}$ and that the fibers of the submersion $\tau \circ \pi$ are the fibers of the submersion $\tau \circ \tilde{\pi}$.

Let $U$ and $V$ be vertical vector fields with respect to $\tau \circ \pi$ and $X$ a horizontal vector field.

We have that $\eta(U)=\eta(V)=0$. Moreover, using (5.1) and the Koszul formula, we deduce that

$$
\tilde{g}\left(\tilde{\nabla}_{U} V, X\right)=g\left(\nabla_{U} V, X\right),
$$

where $\nabla$ and $\tilde{\nabla}$ are the Riemannian connections of the metrics $g$ and $\tilde{g}$ respectively.

Thus, from (8.6) and since the fibers of $\tau \circ \pi$ are totally geodesic submanifolds of $(M, g)$, we obtain that $\tilde{g}\left(\tilde{\nabla}_{U} V, X\right)=0$. Therefore, the fibers of the submersion $\tau \circ \tilde{\pi}$ are totally geodesic submanifolds of $(M, \tilde{g})$.

This ends the proof of our assertion.

Let $c$ be a real number, $c \neq 0$. In corollary 3.2 we show that for all $k>-3 c^{2}$ (respectively $k=-3 c^{2}$ and $k<-3 c^{2}$ ) the total space of the submersion $\pi_{1}(c, k, m-1)$ (respectively $\pi_{2}\left(c, m-1, m^{\prime}\right)$ and $\pi_{3}(c, k, m-1)$ ) is a PC-manifold product of a $c$-Sasakian manifold with the 2-dimensional hyperbolic space $H_{c}^{2}$. In fact, if $(s, t)$ are the coordinates on $H_{c}^{2}$ then the 
submersion $\pi_{1}(c, k, m-1)$ (respectively $\pi_{2}\left(c, m-1, m^{\prime}\right)$ and $\pi_{3}(c, k, m-1)$ ) is a $D(c s)$-conformal cosymplectic submersion with total space a complete simply connected $\mathrm{PC}(k)$-manifold and base space a complete simply connected cosymplectic manifold and such that the fibers are totally geodesic submanifolds.

Next, we shall prove a converse of this corollary, which is the main result of this section.

Let $\pi:(M, \varphi, \xi, \eta, g) \rightarrow\left(M^{\prime}, \varphi^{\prime}, \xi^{\prime}, \eta^{\prime}, g^{\prime}\right)$ a $D(\sigma)$-conformal cosymplectic submersion of the connnected manifold $M$ onto $M^{\prime}$. We shall denote by $\bar{M}$ the universal covering space of $M$, by $(\bar{\varphi}, \bar{\xi}, \bar{\eta}, \bar{g})$ the induced almost contact metric structure on $\bar{M}$ and by $\bar{\pi}:(\bar{M}, \bar{\varphi}, \bar{\xi}, \bar{\eta}, \bar{g}) \rightarrow\left(M^{\prime}, \varphi^{\prime}, \xi^{\prime}, \eta^{\prime}, g^{\prime}\right)$ the induced $D(\bar{\sigma})$-conformal cosymplectic submersion of $\bar{M}$ onto $M^{\prime}$ (sect. 6 ). We shall suppose that the fibers of $\bar{\pi}$ are connected submanifolds of $\bar{M}$.

Theorem 8.1. - Let $\pi:(M, \varphi, \xi, \eta, g) \rightarrow\left(M^{\prime}, \varphi^{\prime}, \xi^{\prime}, \eta^{\prime}, g^{\prime}\right)$ be a $D(\sigma)$ conformal cosymplectic submersion such that $(M, \varphi, \xi, \eta, g)$ is a complete connected $\mathrm{PC}(k)$-manifold with Lee 1-form $\omega$ and the fibers of $\pi$ are totally geodesic submanifolds of $M$. Let $\bar{\pi}:(\bar{M}, \bar{\varphi}, \bar{\xi}, \bar{\eta}, \bar{g}) \rightarrow\left(M^{\prime}, \varphi^{\prime}, \xi^{\prime}, \eta^{\prime}, g^{\prime}\right)$ be the induced $D(\bar{\sigma})$-conformal cosymplectic submersion of the universal covering space $\bar{M}$ of $M$ onto $M^{\prime}$. Suppose $c=\|\omega\|, \operatorname{dim} M=2 m+1$ and $\operatorname{dim} M^{\prime}=2 m^{\prime}+1$.

(1) If $k>-3 c^{2}$ then $\bar{\pi}$ and $\pi_{1}(c, k, m-1)$ are equivalent.

(2) If $k=-3 c^{2}$ then $\bar{\pi}$ and $\pi_{2}\left(c, m-1, m^{\prime}\right)$ are equivalent.

(3) If $k<-3 c^{2}$ then $\bar{\pi}$ and $\pi_{3}(c, k, m-1)$ are equivalent.

Proof. - If $k>-3 c^{2}$ then, by theorem 1.2, we deduce that there exists an almost contact isometry $\tau$ of $(\bar{M}, \bar{\varphi}, \bar{\xi}, \bar{\eta}, \bar{g})$ onto the $\operatorname{PC}(k)$ manifold $\left(S^{2 m-1}(c, k) \times H_{c}^{2}, \tilde{\varphi}, \widetilde{\xi}, \tilde{\eta}, \tilde{g}\right)$, where the almost contact metric structure $(\tilde{\varphi}, \tilde{\xi}, \tilde{\eta}, \tilde{g})$ is given by (1.14). Moreover, if $\tilde{B}$ is the Lee vector field of $S^{2 m-1}(c, k) \times H_{c}^{2}$ and $(s, t)$ the coordinates on $H_{c}^{2}$ then, from corollary 5.2 , we have that $\widetilde{B}$ and $\tilde{\varphi} \widetilde{B}$ are vertical vector fields of the $D(c s)$-conformal cosymplectic submersion $\bar{\pi} \circ \tau^{-1}: S^{2 m-1}(c, k) \times$ $H_{c}^{2} \rightarrow\left(M^{\prime}, \varphi^{\prime}, \xi^{\prime}, \eta^{\prime}, g^{\prime}\right)$. Thus, since the vertical bundle of the submersion $\pi_{1}(c, k, m-1): S^{2 m-1}(c, k) \times H_{c}^{2} \rightarrow P_{m-1}\left(C^{m}\right)\left(k+3 c^{2}\right) \times \mathbb{R}$ is generated by $\widetilde{B}$ and $\widetilde{\varphi} \widetilde{B}$, there exists an almost contact metric submersion $\tau^{\prime}: P_{m-1}\left(C^{m}\right)\left(k+3 c^{2}\right) \times \mathbb{R} \rightarrow\left(M^{\prime}, \varphi^{\prime}, \xi^{\prime}, \eta^{\prime}, g^{\prime}\right)$ such that $\tau^{\prime} \circ \pi_{1}(c, k, m-1)=\bar{\pi} \circ \tau^{-1}$. In fact, $\tau^{\prime}$ is a cosymplectic submersion. 
Almost contact submersions with total space a locally conformal cosymplectic manifold

The following commutative diagram illustrates the above situation.

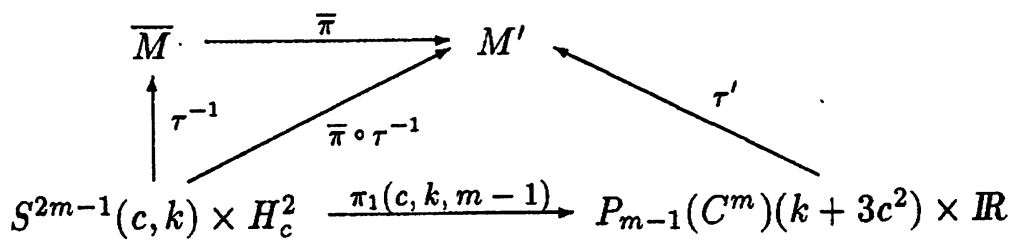

Now, using lemma 8.1, we obtain that $\tau^{\prime}$ is a submersion with connected and totally geodesic fibers which, by proposition 8.3 , implies that $\tau^{\prime}$ is an almost contact isometry. This proves (1).

(3) is proved in a similar way.

Analogously, if $k=-3 c^{2}$, then we deduce that there exists a cosymplectic submersion

$$
\tau^{\prime \prime}: C^{m-1}(0) \times \mathbb{R} \rightarrow\left(M^{\prime}, \varphi^{\prime}, \xi^{\prime}, \eta^{\prime}, g^{\prime}\right)
$$

of $C^{m-1}(0) \times \mathbb{R}$ onto $M^{\prime}$ with totally geodesic fibers and such that $\tau^{\prime \prime} \circ$ $\pi_{2}(c, m-1, m-1)=\bar{\pi} \circ \tau$, being $\tau$ an almost contact isometry of $\mathbb{R}^{2 m-1}(c) \times H_{c}^{2}$ onto $(\bar{M}, \bar{\varphi}, \bar{\xi}, \bar{\eta}, \bar{g})$. Thus, from proposition 8.3 , we have that $\tau^{\prime \prime}$ and the projection $\tau_{2}\left(m-1, m^{\prime}\right)$ of $C^{m-1}(0) \times \mathbb{R}$ onto $C^{m \prime}(0) \times \mathbb{R}$ are equivalent submersions, i.e. the following diagram:

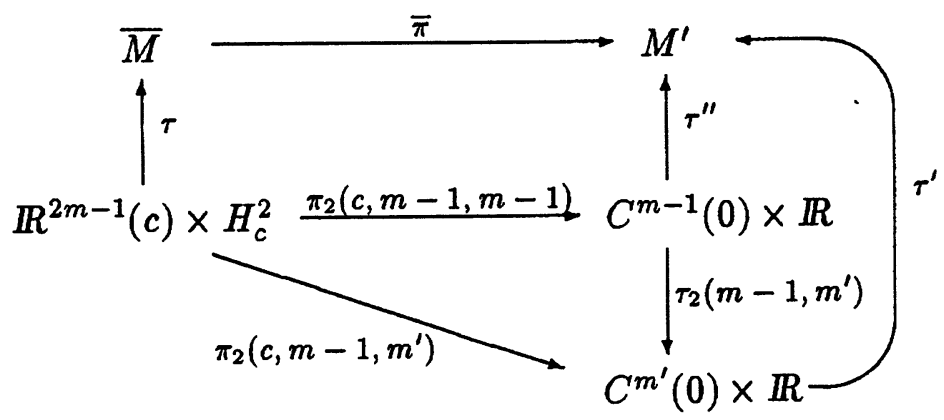

is commutative, where $\tau^{\prime}: C^{m \prime}(0) \times \mathbb{R} \rightarrow M^{\prime}$ is an almost contact isometry. Therefore the $D(\bar{\sigma})$-conformal cosymplectic submersion $\bar{\pi}$ and the $D(c s)$ conformal cosymplectic submersion $\pi_{2}\left(c, m-1, m^{\prime}\right)$ are equivalent. 


\section{References}

[1] Alexiev (V. A.) and Ganchev (G. T.).- Contact conformal transformations of the almost contact metric structures, Comptes rendus de l'Académie Bulgare des Sciences, 39, $\mathrm{n}^{\circ} 9$ (1986), pp. 27-30.

[2] Blair (D. E.) .- Contact manifolds in Riemannian Geometry, Lecture Notes in Math. 509, Springer-Verlag, Berlin, 1976.

[3] Blair (D. E.) and Goldberg (S. I.) .- Topology of almost contact manifolds, J. Diff. Geom. 1 (1967), pp. 347-354.

[4] Capursi (M.) .- Quasi cosymplectic manifolds, Rev. Roumaine Math. Pures Appl. 32, no 1 (1987), pp. 27-35.

[5] Chinea (D.) .- Almost contact metric submersions, Rendiconti del Circlo Mat. di Palermo, Serie II, XXXIV (1985), pp. 89-104.

[6] Chinea (D.) .- Quasi-K-cosymplectic submersions, Rendiconti del Circlo Mat. di Palermo, Serie II, XXXIII (1984), pp. 319-330.

[7] Chinea (D.) and Gonzalez (J. C.) - - A classification of almost contact metric manifolds, Annali di Mat. Pura ed appl. (IV), 156 (1990), pp. 15-36.

[8] Chinea (D.), De León (M.) and Marrero (J. C.) . - Topology of cosymplectic manifolds, Journal de Mathématiques Pures et Appliquées 72 (1993), pp. 567 591.

[9] Chinea (D.) and Marrero (J. C.) - - On invariant submanifolds of locally conformal almost cosymplectic manifolds, Bolletino U.M.I. 4-A, $\mathrm{n}^{\circ} 7$ (1990), pp. 357-364.

[10] Chinea (D.) and Marrero (J. C.) .- Conformal changes of almost contact metric structures, Riv. Mat. Univ. Di Parma 5, n 1 (1992), pp. 19-31.

[11] Chinea (D.) and Marrero (J. C.) - - Conformal changes of almost cosymplectic manifolds, Rendiconti di Matematica, Serie VII, 12 (1992), pp. 849-867.

[12] Dragomir (S.) .- Generalized Hopf manifolds, locally conformal Kähler structures and real hypersurfaces, Kodai Math. J. 14 (1991), pp. 366-391.

[13] EuM (S. S.) .- Cosymplectic manifolds of constant $\varphi$-holomorphic curvature, Bull. Korean Math. Soc. 9 (1972), pp. 1-7.

[14] Janssens (D.) and VANHECKe (L.) .- Almost contact structures and curvature tensors, Kodai Math. J. 4 (1981), pp. 1-27.

[15] КоваYashi (S.) and Nomizu (K.) . - Foundations of Differential Geometry, Vol. I and II, Intersc. Publ., New York (1966 and 1969).

[16] Marrero (J. C.) .- Locally conformal cosymplectic manifolds foliated by generalized Hopf manifolds, Rendiconti di Matematica, Serie VII, 12 (1992), pp. 305327.

[17] Marrero (J. C.) and Rocha (J.) .- Locally conformal Kähler submersions, Geometriae Dedicata 52 (1994), pp. 271-289.

[18] OKUMURA (M.) . - On infinitesimal conformal and projective transformations of normal contact spaces, Tôhoku Math. J. 14 (1962), pp. 398-412. 
Almost contact submersions with total space a locally conformal cosymplectic manifold

[19] O'NeILL (B.) - - The fundamental equations of a submersion, Michigan J. Math. 13 (1966), pp. 459-469.

[20] O'NeILl (B.) .- Semi-Riemannian Geometry with applications to relativity, Academic Press, New York, 1983.

[21] Oubiña (J. A.) - - A classification for almost contact structures, Preprint.

[21'] Rocha (J.) . - Submersiones localmente conforme Kähler y casi contacto con espacio total una variedad localmente conforme cosimpléctica, Tesis Doctoral, Departamento de Matemática Fundamental, Universidad de La Laguna (1995).

[22] Tanno (S.) . - The topology of contact Riemannian manifolds, Mlinois J. Math. 12 (1968), pp. 700-717.

[23] Tanno (S.) .- Sasakian manifolds with constant $\varphi$-holomorphic sectional curvature, Tôhoku Math. J. 21 (1969), pp. 501-507.

[24] De la Torre (E.) .- Inmersiones y submersiones casi hermíticas, Publ. Dpto. Geometría y Topologia 55, Univ. Santiago de Compostela (1982).

[25] Tshikuna-Matamba (T.) .- Quelques classes des submersions métriques presque de contact, Rev. Roumaine Math. Pures Appl. 35 (1990), pp. 705-721.

[26] Vaisman (I.) . - Locally conformal Kähler manifolds with parallel Lee form, Rend. Mat. Roma 12 (1979), pp. 263-284.

[27] Vaisman (I.) .- Conformal changes of almost contact metric structures, Lecture Notes in Math., Springer, 792 (1979), pp. 435-443.

[28] Vaisman (I.) .- Generalized Hopf manifolds, Geometriae Dedicata 13 (1982), pp. 231-255.

[29] Vaisman (I.) and Reischer (C.) .- Local similarity manifolds, Ann. di Mat. Pura ed Appl. (IV), 135 (1983), pp. 279-292.

[30] Watson (B.) .- On almost Hermitian submersions, J. Diff. Geometry 11 (1976), pp. 147-165.

[31] Watson (B.).- The differential geometry of two types of almost contact metric submersions, The Math. Heritage of C. F. Gauss, edited by Georges M. Rassias, 1991 World Scientific Publ. Co. Singapore, pp. 827-861.

[32] YANO (K.) and IshiHARA (S.) .- Invariant submanifolds of an almost contact manifold, Kodai Math. Sem. Rep. 21 (1969), pp. 330-364. 